\title{
Designs esféricos
}

\section{Luiz Carlos Leal Junior}

Orientador: Valdir Antônio menegatto

Dissertação apresentada ao Instituto de Ciências

Matemáticas e de Computação - ICMC-USP, como parte dos requisitos para obtenção do título de Mestre em Matemática.

USP - São Carlos

Agosto/2006 
Á Heliza e ao Caynnan com muito amor. 


\section{Agradecimentos}

Ao professor Valdir Antônio Menegatto, pela orientação, confiança e profissionalismo com que conduziu este trabalho.

A Deus pela ajuda incondicional em todos momentos da minha vida.

À Universidade de São Paulo pela possibilidade de realização deste trabalho.

À CAPES pelo apoio financeiro.

A meus colegas do ICMC-USP, que tornaram esta caminhada mais agradável.

A Francisco e Suelen Granada, que além de serem meu cunhado e minha irmã, são meus grandes amigos e me ajudaram muito contribuindo para mais esta realização.

Ao meu irmão Jhonatta, meus avós, tios, sogros e familiares em geral que tanto me apoiaram neste intento.

Mais que agradecer, eu também dedico este trabalho aos meus pais: Luiz e Roseli. Pelo incentivo, encorajamento e, principalmente, pela oportunidade de estudar e completar mais esta etapa em minha vida. Se cheguei até aqui, foi graças a eles.

Aos meus amores Heliza e Caynnan, pelo apoio, amor e dedicação a mim dispensados. Pelas horas que passei distante deles e pela compreensão, pois foram de vital importância para realização de mais este sonho.

Muito obrigado! 


\begin{abstract}
In this work we will study specials subsets of the unitary sphere $S^{m-1}$ of $\mathbb{R}^{m}, m \geq 2$, usually called in literature spherical designs. The main objectives are to analyze many equivalences for the concept, its connections with both, rotations on $S^{m-1}$ and embedded in spheres of higher dimension, and results on the cardinality of spherical designs.
\end{abstract}




\section{Resumo}

Neste trabalho estudaremos subconjuntos especiais da esfera unitária $S^{m-1}$ de $\mathbb{R}^{m}, m \geq$ 2, comumente chamados na literatura de designs esféricos. Os objetivos principais são analisar várias equivalências para o conceito, suas conexões com ambos, rotações sobre $S^{m-1}$ e mergulhos em esferas de dimensão superior, e resultados sobre a cardinalidade dos designs esféricos. 


\section{Sumário}

Introdução

1 Conceitos básicos $\quad 3$

1.1 Harmônicos esféricos . . . . . . . . . . . . . . . . . . . . 3

1.2 Harmônicos zonais . . . . . . . . . . . . . . . . . . . . 6

1.3 Teorema da adição . . . . . . . . . . . . . . . . . . . 6

1.4 Representação de harmônicos esféricos . . . . . . . . . . . . . . . . . 7

1.5 Fórmula de Funk-Hecke . . . . . . . . . . . . . . . . . . . . 8

1.6 Polinômios de Legendre e a fórmula de Dougall . . . . . . . . . . . . . . . 9

1.7 Núcleos de reprodução . . . . . . . . . . . . . . . . . . . . . 12

2 Designs esféricos $\quad 15$

2.1 Designs esféricos . . . . . . . . . . . . . . . . . 15

2.2 Designs esféricos e invariância . . . . . . . . . . . . . . . . 20

2.3 Designs esféricos via formas quadráticas . . . . . . . . . . . . . 22

2.4 Resultados Adicionais . . . . . . . . . . . . . . . . . 26

2.5 Uma aplicação . . . . . . . . . . . . . . . . . . . . . . . 28

3 Designs esféricos de cardinalidade mínima 31

4 Rotações de Designs esféricos $\quad 37$

$\begin{array}{ll}\text { Apêndice } 1 & 41\end{array}$

$\begin{array}{ll}\text { Notações } & 43\end{array}$ 


\section{Introdução}

A origem do termo "design esférico" data de 1977 quando P. Delsarte, J. M. Goethals e J. J. Seidel publicaram seu mais famoso trabalho [8]. Eles introduziram os designs esféricos quando estudando códigos esféricos. Estes aparecem em conexão com o problema de distribuir-se uma quantidade finita de pontos em $S^{m-1}$, maximizando a menor distância entre eles. Mais detalhes sobre os códigos esféricos podem ser obtidos fazendo-se uma pesquisa no google usando como palavra chave spherical code.

A idéia principal por trás do conceito de design esférico talvez seja aquela de buscar subconjuntos finitos de $S^{m-1}$ para manipular integração numérica em $S^{m-1}$ utilizando pesos iguais. A formulação conceitual que apresentaremos é aquela que traduz a possibilidade de calcularmos o valor médio sobre $S^{m-1}$ de qualquer polinômio com grau pré-determinado, conhecendo-se os valores do polinômio em um subconjunto finito de pontos de $S^{m-1}$. Um procedimento de pesquisa similar ao indicado no parágrafo anterior, mas usando como palavra chave "spherical design", produz uma farta lista de sites com informações sobre o tema aqui considerado. Em particular, a página de R. Womersley é bem ilustrativa.

No Capítulo 1, apresentamos alguns resultados preliminares sobre a análise na esfera. Isto inclui alguns sobre polinômios em $S^{m-1}$, incluindo polinômios homogêneos e harmônicos esféricos. Introduzimos os espaços básicos que são utilizados, bem como suas estruturas. Analisamos ainda: a invariância de algumas integrais na esfera por transformações ortogonais de $\mathbb{R}^{m}$, harmônicos zonais, a Fórmula de Adição para harmônicos esféricos, a decomposição de $L^{2}\left(S^{m-1}\right)$ como soma ortogonal de espaços de harmônicos esféricos, etc. Finalmente, consideramos a fórmula de Funk-Hecke, os polinômios de Legendre e alguns resultados sobre núcelos de reprodução.

No Capítulo 2, iniciamos com o conceito de design esférico e alguns exemplos preliminares na Seção 2.1. Na Seção 2.2, apresentamos mais equivalências para o conceito 
de design esférico, agora usando idéias de invariância. Na Seção 2.3, utilizamos idéias de positividade definida para discutir três equivalências adicionais para então apresentar no Teorema 2.3.2 as equivalências mais econômicas, pelo menos do ponto de vista de verificação, do conceito. A útlima equivalência da seção é puramente técnica. A Seção 2.4 apresenta alguns resultados adicionais enquanto que a Seção 2.5 inclui uma aplicação dos resultados na contrução de designs esféricos em esferas de dimensões diferentes.

No Capítulo 3, fazemos uma ampla discussão sobre os limitantes inferior e superior para a cardinalidade de um design esférico.

O Capítulo 4 contém resultados parecidos com aquele apresentado na Seção 2.5.

No Apêndice indicamos rapidamente como deve ser feita a escolha de índices quando da aplicação do Teorem 2.4.2 na prova de resultados do Capítulo 3.

Em seguida fornecemos uma tabela com as notações utilizadas no trabalho.

Concluímos, com uma lista de referências bibliográficas utilizadas no desenvolvimento deste trabalho. 


\section{Capítulo 1}

\section{Conceitos básicos}

Neste capítulo pretendemos apresentar definições e resultados que possibilitam a fundamentação teórica para os designs esféricos. Os resultados aqui expostos são de certa forma clássicos na teoria de análise na esfera e podem ser encontrados nas referêencias $[2,11,21]$. Por este motivo, algumas demonstrações de teoremas e proposições não são incluídas.

\subsection{Harmônicos esféricos}

Seja $n$ um inteiro não negativo. Uma função $f: \mathbb{R}^{m} \rightarrow \mathbb{R}$ é dita ser homogênea de grau $n$ se

$$
f(\lambda x)=\lambda^{n} f(x), \quad \lambda>0, \quad x \in \mathbb{R}^{m} .
$$

Ela é dita ser harmônica quando

$$
\sum_{j=1}^{m} \frac{\partial^{2} f}{\partial x_{j}^{2}} \equiv 0, \quad x=\left(x_{1}, x_{2}, \ldots, x_{m}\right) .
$$

Definição 1.1.1. Um polinômio esférico de grau $n$ em $m$ variáveis é a restrição de um polinômio de grau $n$ em $m$ variáveis à $S^{m-1}$.

Definição 1.1.2. Um harmônico esférico sólido de dimensão $m$ e grau n é um polinômio homogêneo de grau $n$ em $m$ variáveis que é harmônico. A restrição de um harmônico

esférico sólido de dimensão $m$ e grau $n$ à $S^{m-1}$ é um harmônico esférico de dimensão $m$ e grau $n$. 
Denotaremos por $\mathcal{P}_{n}$ o conjunto formado pelos elementos de $\Pi_{n}$ restritos à $S^{m-1}$, por $\mathcal{H}_{n}$ o subconjunto de $\mathcal{P}_{n}$ formado pelos polinômios que são homogêneos, e por $\mathcal{A}_{n} \mathrm{o}$ subconjunto de $\mathcal{P}_{n}$ formado pelos harmônicos esféricos de grau $n$.

Usaremos a estrutura do espaço $L^{2}\left(S^{m-1}\right)$ usual. A expressão

$$
\langle f, g\rangle=\int_{S^{m-1}} f(x) g(x) d \sigma(x), \quad f, g \in L^{2}\left(S^{m-1}\right),
$$

define um produto interno nesse espaço, onde $\sigma_{m-1}$ é a medida de superfície usual sobre $S^{m-1}$. A menos de qualquer especificação em contrário, ortogonalidade e ortonormalidade referir-se-ão ao produto interno (1.1).

O teorema a seguir trata da invariância da medida $\sigma_{m-1}$ por elementos de $\mathcal{O}_{m}$.

Teorema 1.1.1. Sejam $f \in L^{2}\left(S^{m-1}\right)$ e $\rho \in \mathcal{O}_{m}$. Então $f \circ \rho$ é $\sigma_{m-1}$-integrável $e$

$$
\int_{S^{m-1}} f(\rho(x)) d \sigma(x)=\int_{S^{m-1}} f(x) d \sigma(x) .
$$

Em particular, se $g, h \in L^{2}\left(S^{m-1}\right)$, então

$$
\langle g \circ \rho, h \circ \rho\rangle=\langle g, h\rangle, \quad \rho \in \mathcal{O}_{m} .
$$

A demonstração deste resultado pode ser encontrada em ([11], p.13).

A seguir, listamos alguns resultados sobre a ortogonalidade dos espaços $\mathcal{A}_{n}$, a decomposição de polinômios esféricos como combinações lineares de polinômios harmônicos e as dimensões dos espaços $\mathcal{A}_{n}$ e $\mathcal{H}_{n}$.

Os dois resultados a seguir sobre a ortogonalidade nos espaços $\mathcal{A}_{n}$ podem ser encontrados em ([11], p.68).

Teorema 1.1.2. Se $l \neq k$, então $\mathcal{A}_{l}$ e $\mathcal{A}_{k}$ são subespaços ortogonais.

Corolário 1.1.1. Se $h \in \mathcal{A}_{n}$ e $n \neq 0$, então

$$
\int_{S^{m-1}} h(x) d \sigma(x)=0 .
$$

A decomposição básica de um polinômio homogêneo como soma de polinômios harmônicos é dada no teorema abaixo ([11], p.69).

Teorema 1.1.3. Seja p um elemento de $\mathcal{H}_{n}$. Então, existe uma única decomposição na forma

$$
p=\sum_{j=0}^{l} h_{n-2 j}, \quad l=\lfloor n / 2\rfloor,
$$

onde $h_{n-2 j} \in \mathcal{A}_{n-2 j}, j=0,1, \ldots, l$. 
Os espaços $\mathcal{A}_{n}$ e $\mathcal{H}_{n}$ são de dimensão finita e suas dimensões serão denotadas por $N_{n}$ e $\tau_{n}$, respectivamente. Em particular, $\tau_{n}=\sum_{j=0}^{l} N_{n-2 j}$.

Proposição 1.1.1. Vale a fórmula

$$
N_{n}= \begin{cases}1, & \text { se } n=0 \\ (m+2 n-2) \frac{(n+m-3) !}{n !(m-2) !}, & \text { se } n \in \mathbb{N} .\end{cases}
$$

Proposição 1.1.2. Se $n$ é um inteiro não negativo, então $\mathcal{P}_{n}=\bigoplus_{i=0}^{n} \mathcal{A}_{i}$.

Como

$$
N_{n}=\left(\begin{array}{c}
m+n-1 \\
m-1
\end{array}\right)-\left(\begin{array}{c}
m+n-3 \\
m-1
\end{array}\right), \quad n \geq 1,
$$

a proposição anterior fornece a seguinte decomposição para a dimensão $d_{n}$ do espaço $\mathcal{P}_{n}$ :

$$
d_{n}=\sum_{l=0}^{n} N_{l}, \quad n \geq 1
$$

Usando a propriedade do fatorial podemos ver que

$$
d_{n}=\left(\begin{array}{c}
m+n-1 \\
m-1
\end{array}\right)+\left(\begin{array}{c}
m+n-2 \\
m-1
\end{array}\right) .
$$

Segue da Proposição 1.1.2 que existe uma base ortonormal de $\mathcal{P}_{n}$ na forma

$$
\cup_{k=0}^{n}\left\{Y_{1}^{k}, Y_{2}^{k}, \ldots, Y_{N_{k}}^{k}\right\}
$$

onde $\left\{Y_{1}^{k}, Y_{2}^{k}, \ldots, Y_{N_{k}}^{k}\right\}$ é uma base ortonormal de $\mathcal{A}_{k}$. Fazendo $n$ crescer indefinidamente obtemos então uma base ortonormal correspondente para $L^{2}\left(S^{m-1}\right)$. Qualquer base ortonormal fixa considerada nas seções subseqüentes é assumida ser da forma acima.

Proposição 1.1.3. $\left[\cup_{i=0}^{\infty} \mathcal{A}_{i}\right]$ é denso em $L^{2}\left(S^{m-1}\right)$.

Prova: Veja [2], p.81.

O próximo teorema estabelece a invariância de $\mathcal{A}_{n}$ por elementos de $\mathcal{O}_{m}$. Sua prova pode ser encontrada em [11], p.69.

Teorema 1.1.4. As seguintes afirmações valem:

(i) Se $h \in \mathcal{A}_{n}$ e $\rho \in \mathcal{O}_{m}$, então $h \circ \rho \in \mathcal{A}_{n}$;

(ii) Se $\left\{h_{1}, h_{2}, \ldots, h_{N_{n}}\right\}$ é uma base ortonormal de $\mathcal{A}_{n}$, então $\left\{h_{1} \circ \rho, h_{2} \circ \rho, \ldots, h_{N_{n}} \circ \rho\right\}$ é uma base ortonormal de $\mathcal{A}_{n}$. 


\subsection{Harmônicos zonais}

Definição 1.2.1. Seja $\varepsilon$ um ponto de $S^{m-1}$. Uma função $f: S^{m-1} \rightarrow \mathbb{R}$ é dita ser $\varepsilon$-zonal $s e$

$$
f(x)=g(x \cdot \varepsilon), \quad x \in S^{m-1}
$$

para alguma $g:[-1,1] \rightarrow \mathbb{R}$.

O Teorema 1.2.1 abaixo apresenta duas propriedades dos harmônicos esféricos que são zonais. Para uma prova detalhada veja [11], p.80.

Teorema 1.2.1. Seja $Z_{\varepsilon}^{n}$ um harmônico esférico $\varepsilon$-zonal. Valem as seguintes propriedades: (i) $S e\left\{Y_{1}^{n}, Y_{2}^{n}, \ldots, Y_{N_{n}}^{n}\right\}$ é uma base ortonormal de $\mathcal{A}_{n}$, então

$$
Z_{\varepsilon}^{n}(x)=\sum_{j=1}^{N_{n}} Y_{j}^{n}(x) Y_{j}^{n}(\varepsilon), \quad x \in S^{m-1} .
$$

(ii) Se $\rho \in \mathcal{O}(m)$ é tal que $\rho(\varepsilon)=\varepsilon$, então $Z_{\varepsilon}^{n} \circ \rho=Z_{\varepsilon}^{n}$.

\subsection{Teorema da adição}

O teorema da adição, uma generalização da fórmula da adição para polinômios trigonométricos, é um resultado clássico dentro da análise esférica. Ela faz a ponte entre ortogonalidade em $L^{2}\left(S^{m-1}\right)$ e ortogonalidade para funções definidas em $[-1,1]$ de uma maneira quase natural. A referência básica para a formulação que aqui apresentamos pode ser encontrada em ([14], p.27).

Teorema 1.3.1 (Fórmula da adição). Vale a fórmula

$$
\sum_{j=1}^{N_{n}} Y_{j}^{n}(x) Y_{j}^{n}(y)=\frac{N_{n}}{\sigma_{m-1}} R_{n}^{m}(x \cdot y), \quad x, y \in S^{m-1},
$$

onde $R_{n}^{m}$ é o polinômio de Legendre de grau $n$ associado à dimensão $m$.

Corolário 1.3.1. Seja $W$ um subconjunto finito e não-vazio de $S^{m-1}$. A forma quadrática $\sum_{x \in W} \sum_{y \in W} c_{x} c_{y} R_{n}^{m}(x \cdot y)$ é não negativa definida, com $c_{x}$ e $c_{y}$ constantes.

Prova: Isto segue da igualdade

$$
\sum_{x \in W} \sum_{y \in W} c_{x} c_{y} R_{n}^{m}(x \cdot y)=\frac{\sigma_{m-1}}{N_{n}} \sum_{j=1}^{N_{n}}\left(\sum_{x \in W} c_{x} Y_{j}^{n}(x)\right)^{2} \geq 0,
$$


uma conseqüencia da Fórmula da adição.

Os polinômios de Legendre são polinômios normalizados pela relação $R_{n}^{m}(1)=1$ ([11], pg. 82). Quando $m \geq 3$, temos a seguinte fórmula de recorrência

$$
R_{n}^{m}=\frac{(m-3) ! n !}{(n+m-3) !} P_{n}^{(m-2) / 2}
$$

onde $P_{n}^{(m-2) / 2}$ é o polinômio de Gegenbauer de grau $n$ associado à dimensão $(m-2) / 2$ $[11,17,22]$.

Os polinômios de Gegenbauer $P_{n}^{\lambda}, \lambda>-1 / 2$, são gerados pela série

$$
\sum_{l=0}^{\infty} P_{i}^{\lambda}(t) x^{i}=\frac{1}{\left(1-2 t x+x^{2}\right)^{\lambda}}, \quad t \in[-1,1],
$$

são ortogonais em relação ao produto interno

$$
\langle f, g\rangle^{\prime}=\int_{-1}^{1} f(t) g(t)\left(1-t^{2}\right)^{(m-3) / 2} d t .
$$

e são casos particulares dos polinômios de Jacobi. Precisamente,

$$
P_{n}^{\lambda}=\frac{(2 \lambda)_{n}}{(\lambda+1 / 2)_{n}} P_{n}^{(\lambda-1 / 2, \lambda-1 / 2)}
$$

onde

$$
(\lambda)_{n}=\prod_{j=1}^{n}(\lambda+j-1), \quad n \geq 1, \quad(\lambda)_{0}=1, \quad \lambda \neq 0,
$$

e $P_{n}^{(\alpha, \beta)}$ é o polinômio de Jacobi de grau $n$ associado aos índices $\alpha$ e $\beta$.

Concluimos esta seção registrando a fórmula de recorrência para polinômios de Legendre ([11], p.89).

Proposição 1.3.1. Os polinômios de Legendre satisfazem a seguinte relação de recorrência

$$
(n+m-2) R_{n+1}^{m}(t)=(2 n+m-2) t R_{n}^{m}(t)-n R_{n-1}^{m}(t), \quad n \in \mathbb{N}, \quad t \in[-1,1] .
$$

\subsection{Representação de harmônicos esféricos}

Vamos agora procurar uma representação para os harmônicos esféricos em termos dos polinômios de Legendre.

Definição 1.4.1. Seja $V$ um espaço d-dimensional de funções definidas em $S^{m-1}$. Um subconjunto $\left\{x_{1}, x_{2}, \ldots, x_{d}\right\}$ de $S^{m-1}$ é chamado um sistema fundamental para $V$ quando a matriz com entradas $f_{j}\left(x_{i}\right)$ é invertível qualquer que seja a base $\left\{f_{1}, f_{2}, \ldots, f_{d}\right\}$. 
Proposição 1.4.1. Seja $k$ um inteiro não negativo. Então é possível encontrar um sistema fundamental para $\mathcal{A}_{k}$.

Prova: Seja $\left\{Y_{1}^{k}, Y_{2}^{k}, \ldots, Y_{N_{k}}^{k}\right\}$ uma base ortogonal de $\mathcal{A}_{k}$. Certamente podemos encontrar um ponto $x_{1} \in S^{m-1}$ tal que $Y_{1}^{k}\left(x_{1}\right) \neq 0$. Como o conjunto $\left\{Y_{1}^{k}, Y_{2}^{k}\right\}$ é linearmente independente, existe $x_{2} \in S^{m-1}$ tal que

$$
\left|\begin{array}{cc}
Y_{1}^{k}\left(x_{1}\right) & Y_{1}^{k}\left(x_{2}\right) \\
Y_{2}^{k}\left(x_{1}\right) & Y_{2}^{k}\left(x_{2}\right)
\end{array}\right| \neq 0
$$

O resto segue indutivamente.

Corolário 1.4.1. Seja $\left\{x_{1}, x_{2}, \ldots, x_{N_{k}}\right\}$ um sistema fundamental para $\mathcal{A}_{k}$. Então, qualquer $h \in \mathcal{A}_{k}$ pode ser escrita na forma

$$
h(\xi)=\sum_{j=1}^{N_{k}} a_{j} R_{k}^{m}\left(\xi \cdot x_{j}\right), \quad \xi \in S^{m-1}, \quad a_{j} \in \mathbb{R}, \quad j=1,2, \ldots, N_{k} .
$$

Prova: Decorre da fórmula da adição que

$$
R_{k}^{m}\left(x_{i} \cdot \xi\right)=\frac{\sigma_{m-1}}{N_{k}} \sum_{j=1}^{N_{k}} Y_{j}^{k}\left(x_{i}\right) Y_{j}^{k}(\xi), \quad \xi \in S^{m-1}, \quad i=1,2, \ldots, N_{k}
$$

ou seja,

$$
\left(\begin{array}{c}
R_{k}^{m}\left(x_{1} \cdot \xi\right) \\
\vdots \\
R_{k}^{m}\left(x_{N_{k}} \cdot \xi\right)
\end{array}\right)=\frac{\sigma_{m-1}}{N_{k}} A^{*}\left(\begin{array}{c}
Y_{1}^{k}(\xi) \\
\vdots \\
Y_{N_{k}}^{k}(\xi)
\end{array}\right), \quad \xi \in S^{m-1}
$$

onde $A=\left(Y_{i}^{k}\left(x_{j}\right)\right)$. Como $A$ é invertível pelo teorema anterior, segue que cada $Y_{j}^{k}$ é combinação linear dos elementos do conjunto $\left\{R_{k}^{m}\left(x_{j} \cdot \xi\right): j=1,2, \ldots, N_{k}\right\}$. O resultado segue.

\subsection{Fórmula de Funk-Hecke}

A seguir apresentamos um dos resultados mais antigos da análise esférica. De uma maneira geral ele diz que uma função da forma $(x, y) \in S^{m-1} \times S^{m-1} \rightarrow K(x \cdot y)$ é um núcleo de reprodução para os espaços $\mathcal{A}_{k}$. Referências para este teorema são $[11,2]$.

No enunciado do teorema usamos o símbolo $L_{\lambda}$ para denotar o conjunto das funções $K:[-1,1] \rightarrow \mathbb{R}$ que satisfazem

$$
\int_{-1}^{1}|K(t)|\left(1-t^{2}\right)^{\lambda-1 / 2} d t<\infty
$$


Teorema 1.5.1. Se $K \in L_{(m-2) / 2}$ e $h \in \mathcal{A}_{k}$, então

$$
\int_{S^{m-1}} K(x \cdot y) h(y) d \sigma(y)=\alpha_{k}(K) h(x), \quad x \in S^{m-1}
$$

onde

$$
\alpha_{k}(K):=\sigma_{m-2} \int_{-1}^{1} K(t) R_{k}^{m}(t)\left(1-t^{2}\right)^{(m-3) / 2} d t .
$$

Utilizaremos o teorema acima $\operatorname{com} K=R_{k}^{m}$.

Corolário 1.5.1. Se $h \in \mathcal{A}_{k}$, então

$$
h(x)=\frac{N_{k}}{\sigma_{m-1}} \int_{S^{m-1}} R_{k}^{m}(x \cdot y) h(y) d \sigma(y), \quad x \in S^{m-1} .
$$

Prova: Substituindo $K=R_{k}^{m}$ em (1.12) e calculando o coeficiente $\alpha_{k}(K)$ correspondente, vem que

$$
\alpha_{k}(K)=\sigma_{m-2} \int_{-1}^{1} R_{k}^{m}(t)^{2}\left(1-t^{2}\right)^{(m-3) / 2} d t=\int_{S^{m-1}}\left|R_{k}^{m}(x \cdot y)\right|^{2} d \sigma(y), \quad x \in S^{m-1} .
$$

Entretanto, pelo Teorema 1.3.1 sabemos que

$$
\left\|R_{k}^{m}(x \cdot \star)\right\|_{2}^{2}=\left(\frac{\sigma_{m-1}}{N_{k}}\right)^{2} \sum_{j=1}^{N_{k}}\left|Y_{j}^{k}(x)\right|^{2}=\frac{\sigma_{m-1}}{N_{k}} .
$$

Logo,

$$
\alpha_{k}(K)=\sigma_{m-1} / N_{k}
$$

e o resultado segue.

\subsection{Polinômios de Legendre e a fórmula de Dougall}

Nesta seção apresentamos algumas propriedades específicas dos polinômios $R_{n}^{m}$.

Lema 1.6.1. Sejam $n$ e $k$ inteiros não negativos. Então,

$$
\left\langle R_{n}^{m}(x \cdot \star), R_{k}^{m}(y \cdot \star)\right\rangle=\frac{\sigma_{m-1}}{N_{n}} R_{n}^{m}(x, y), \quad x, y \in S^{m-1} .
$$

Prova: A fórmula da adição implica que

$$
\begin{aligned}
\left\langle R_{n}^{m}(x \cdot \star), R_{k}^{m}(y \cdot \star)\right\rangle & =\int_{S^{m-1}} R_{n}^{m}(x \cdot z), R_{k}^{m}(y \cdot z) d \sigma(z) \\
& =\sum_{i=1}^{N_{n}} \sum_{j=1}^{N_{k}} \frac{\sigma_{m-1}^{2}}{N_{n} N_{k}} Y_{i}^{n}(x) Y_{j}^{k}(y) \int_{\sigma_{m-1}} Y_{i}^{n}(z) Y_{j}^{k}(z) d \sigma(z) .
\end{aligned}
$$


Por outro lado, a ortogonalidade dos harmônicos esféricos implica que

$$
\begin{aligned}
\sum_{i=1}^{N_{n}} \sum_{j=1}^{N_{k}} \frac{\sigma_{m-1}^{2}}{N_{n} N_{k}} Y_{i}^{n}(x) Y_{j}^{k}(y) \int_{\sigma_{m-1}} Y_{i}^{n}(z) Y_{j}^{k}(z) d \sigma(z) & =\sum_{i=1}^{N_{n}} \sum_{j=1}^{N_{k}} \frac{\sigma_{m-1}^{2}}{N_{n} N_{k}} Y_{i}^{n}(x) Y_{j}^{k}(y) \delta_{n, k} \delta_{i, j} \\
& =\sum_{i=1}^{N_{n}}\left(\frac{\sigma_{m-1}}{N_{n}}\right)^{2} Y_{i}^{n}(x) Y_{i}^{n}(y) \\
& =\frac{\sigma_{m-1}}{N_{n}} R_{n}^{m}(x \cdot y) .
\end{aligned}
$$

Isso completa a prova do teorema.

O lema abaixo enfatiza que os coeficientes na decomposição de monômios como combinação linear de polinômios de Legendre são todos positivos quando o grau do polinômio e do monômio têm a mesma paridade. Uma demonstração formal desse resultado pode ser encontrada em [4].

Lema 1.6.2. Seja $k$ um inteiro não negativo. Então

$$
t^{k}=\sum_{j=0}^{\lfloor k / 2\rfloor} c_{k-2 j} R_{k-2 j}^{m}(t), \quad-1 \leq t \leq 1,
$$

onde $c_{k-2 j}>0, j=0,1, \ldots,\lfloor k / 2\rfloor$.

Os próximos teoremas especificam a ortogonalidade mencionada quando da introdução do produto interno (1.7) ([11], p.83).

Teorema 1.6.1. Se $k$ e $n$ são inteiros não negativos, então

$$
\left\langle R_{k}^{m}, R_{n}^{m}\right\rangle^{\prime}=\delta_{k, n} \frac{\sigma_{m-1}}{\sigma_{m-2} N_{k}}
$$

Teorema 1.6.2. Se $\left\{G_{i}: i \in I \subset \mathbb{N}\right\}$ é uma família (finita ou infinita) de polinômios definidos em $[-1,1]$ tais que $G_{k}$ tem grau $k$, e que para $k \neq l\left\langle G_{l}, G_{k}\right\rangle^{\prime}=0$, então

$$
G_{i}(t)=\omega_{i}^{m} R_{i}^{m}(t), \quad i \in I, \quad t \in[-1,1],
$$

onde $\omega_{i}^{m}$ é uma constante que não depende de $t$.

Finalizamos esta seção enunciando a fórmula de adição para polinômios de Gegenbauer, provada por Gegenbauer em 1875. Não encontramos na literatura a versão desta fórmula para polinômios de Legendre. No entanto, no único ponto desse trabalho onde a fórmula é necessária, a versão aqui apresentada é mais que o suficiente. 
Teorema 1.6.3. Sejam $\theta, \phi$ e $\vartheta$ números reais. Então

$$
\begin{aligned}
P_{n}^{(m-2) / 2}(\cos \theta \cos \phi+\sin \theta \operatorname{sen} \phi \cos \vartheta) & =P_{n}^{(m-2) / 2}(\cos \theta) P_{n}^{(m-2) / 2}(\cos \phi) \\
& +\sum_{i=1}^{n} \beta_{i, n} Q_{i}(\theta) Q_{i}(\phi) P_{i}^{(m-3) / 2}(\cos \vartheta)
\end{aligned}
$$

onde $\beta_{i, n}>0, i=1,2, \ldots, n e$

$$
Q_{i}(\theta)=(\operatorname{sen} \theta)^{i} P_{n-i}^{(m-2+2 i) / 2}(\cos \theta), \quad i=1,2, \ldots, n, \quad \theta \in \mathbb{R} .
$$

Prova: Veja ([1], p.30).

A fórmula de Dougall é uma generalização da fórmula de adição para cossenos

$$
2 \cos l t \cos k t=\cos (k-l) t+\cos (k+l) t
$$

Uma de suas formulações envolve a seguinte relação de ortogonalidade:

$$
\int_{-1}^{1} R_{k}^{m}(t) R_{l}^{m}(t) R_{n}^{m}(t)\left(1-t^{2}\right)^{(m-3) / 2} d t=a_{k, l, n} \int_{-1}^{1}\left(R_{\mu}^{m}(t)\right)^{2}\left(1-t^{2}\right)^{(m-3) / 2} d t .
$$

Aqui, $2 \mu:=k+l+n$ e $a_{k, l, n}$ é um número real positivo.

Teorema 1.6.4. Sejam $k$ e $n$ inteiros não negativos. Então

$$
R_{k}^{m} R_{n}^{m}=\sum_{j=0}^{k \wedge n} \alpha_{j}^{k, n} R_{k+n-2 j}^{m}
$$

onde cada $\alpha_{j}^{k, n}$ é positivo.

Demonstrações deste teorema podem ser encontradas em [12, 7] e ([1], p. 39).

O seguinte lema técnico é uma consequência da fórmula de Funk-Hecke.

Lema 1.6.3. Seja $t \in[-1,1]$. Então

$$
\int_{-1}^{1}\left(1-t^{2}\right)^{(m-3) / 2} d t=\frac{\sigma_{m-1}}{\sigma_{m-2}}
$$

A seguir, vamos calcular um dos coeficientes na fórmula de Dougall, para uso posterior.

Proposição 1.6.1. Vale a seguinte fórmula: $\alpha_{k}^{k, k}=N_{k}^{-1}, k \in \mathbb{N}$.

Prova: Tomando $n=k$ em (1.17) obtemos

$$
R_{k}^{m} R_{k}^{m}=\sum_{j=0}^{k} \alpha_{j}^{k, k} R_{2 k-2 j}^{m}
$$


Integrando ambos os lados de (1.18) e utilizando o Teorema 1.6.1 e o Lema 1.6.3, obtemos

$$
\begin{aligned}
\int_{-1}^{1}\left(R_{k}^{m}(t)\right)^{2}\left(1-t^{2}\right)^{(m-3) / 2} d t & =\sum_{j=0}^{k} \alpha_{j}^{k, k} \int_{-1}^{1} R_{2 k-2 j}^{m}(t)\left(1-t^{2}\right)^{(m-3) / 2} d t \\
& =\alpha_{k}^{k, k} \int_{-1}^{1} R_{0}^{m}(t)\left(1-t^{2}\right)^{(m-3) / 2} d t \\
& =\alpha_{k}^{k, k} \int_{-1}^{1}\left(1-t^{2}\right)^{(m-3) / 2} d t=\alpha_{k}^{k, k} \frac{\sigma_{m-1}}{\sigma_{m-2}}
\end{aligned}
$$

Por outro lado, usando o Teorema 1.6.1 obtemos a seguinte igualdade

$$
\int_{-1}^{1}\left(R_{k}^{m}(t)\right)^{2}\left(1-t^{2}\right)^{(m-3) / 2} d t=\frac{\sigma_{m-1}}{\sigma_{m-2} N_{k}}
$$

e o resultado segue.

\subsection{Núcleos de reprodução}

O termo núcleos de reprodução refere-se geralmente a espaços de Hilbert construídos a partir de funções positivas definidas ou afins. Sua teoria tem muitas utilidades em Teoria da Aproximação e Análise em geral. Neste trabalho, eles entram principalmente no Capítulo 3, quando analisamos limitantes para a cardinalidade de um design esférico.

Definição 1.7.1. Seja $V$ um espaço de Hilbert de funções com domínio D. Uma função $\Phi: D \times D \mapsto \mathbb{R}$ é um núcleo de reprodução de $V$ se

i) Fixado $y \in D$, a função $x \in D \rightarrow \Phi(x, y)$ é um elemento de $V$.

ii) Fixado $y \in D$,

$$
f(y)=[f, \Phi(\star, y)], \quad f \in V,
$$

onde $[\star, \star]$ denota o produto interno de $V$.

Se $V$ tem dimensão finita, não é difícil verificar que $V$ possui um único núcleo de reprodução ([19], p.4).

No restante desta seção, descrevemos os núcleos de reprodução de alguns dos espaços descritos nas seções anteriores.

No que segue vamos utilizar as funções

$$
\mathcal{G}_{n}:=\sum_{j=0}^{n} N_{j} R_{j}^{m}, \quad n=0,1, \ldots
$$


Teorema 1.7.1. Seja $n$ um inteiro não negativo. Então a função $(x, y) \in S^{m-1} \times S^{m-1} \rightarrow$ $\sigma_{m-1}^{-1} \mathcal{G}_{n}(x \cdot y)$ é um núcleo de reprodução de $\mathcal{P}_{n}$.

Prova: Seja $y \in S^{m-1}$. A fórmula de adição implica que a função $x \in S^{m-1} \rightarrow \sigma_{m-1}^{-1} \mathcal{G}_{n}(x$. $y$ ) é um elemento de $\mathcal{P}_{n}$. Por outro lado,

$$
\begin{aligned}
\left\langle p, \sigma_{m-1}^{-1} \mathcal{G}_{n}(\star \cdot y)\right\rangle & =\left\langle p, \sum_{\mu=0}^{n} \frac{N_{\mu}}{\sigma_{m-1}} R_{\mu}^{m}(\star \cdot y)\right\rangle \\
& =\left\langle p, \sum_{\mu=0}^{n} \sum_{j=1}^{N_{\mu}} Y_{j}^{\mu}(\star) Y_{j}^{\mu}(y)\right\rangle \\
& =\sum_{\mu=0}^{n} \sum_{j=1}^{N_{\mu}}\left\langle p, Y_{j}^{\mu}\right\rangle Y_{j}^{\mu}(y)=p(y), \quad p \in \mathcal{P}_{n},
\end{aligned}
$$

pela linearidade do produto interno.

Vamos agora apresentar o núcleo de reprodução de $\mathcal{A}_{n}$.

Teorema 1.7.2. Seja $n$ um inteiro não negativo. Então a função $(x, y) \in S^{m-1} \times S^{m-1} \rightarrow$ $\sigma_{m-1}^{-1} N_{n} R_{n}^{m}(x \cdot y)$ é um núcleo de reprodução de $\mathcal{A}_{n}$.

Prova: É similar à prova do Teorema 1.7.1. 


\section{Capítulo 2}

\section{Designs esféricos}

Neste capítulo introduzimos formalmente o conceito de designs esféricos, analisando suas várias formulações equivalentes e apresentando alguns exemplos elementares. Enfatizamos que não estamos diretamente interessados na busca de exemplos sofisticados ou mesmo na discussão de aspéctos geométricos dos designs.

\subsection{Designs esféricos}

A definição usual de design esférico exige que fórmulas de quadratura com pesos iguais sejam exatas para todos os polinômios esféricos até um determinado grau. Precisamente, temos:

Definição 2.1.1. Um subconjunto finito e não-vazio $W$ de $S^{m-1}$ é um q-design esférico quando

$$
\frac{1}{\sigma_{m-1}} \int_{S^{m-1}} f(x) d \sigma(x)=\frac{1}{|W|} \sum_{x \in W} f(x), \quad f \in \mathcal{P}_{q} .
$$

Observação 2.1.1. Segue da Proposição 1.1.2 que um subconjunto $W$ de $S^{m-1}$ é um q-design se, e somente se, a Fórmula (2.1) vale quando $f \in \cup_{j=0}^{q} \mathcal{A}_{j}$.

Exemplo 2.1.1. Um subconjunto não-vazio $W$ de $S^{m-1}$ é um 1-design se e somente se $\sum_{x \in W} x=0$.

Exemplo 2.1.2. Se $W \subset S^{m-1}$ é um q-design e $q_{1} \in\{0,1, \ldots, q\}$ então $W$ é um $q_{1}$ design. 
Os próximos resultados apresentam várias equivalências para o conceito acima. Devido aos exemplos acima, no que segue, $q$ será um inteiro no mínimo 2.

Teorema 2.1.1. Seja $W$ um subconjunto finito e não-vazio de $S^{m-1}$. Então as seguintes afirmações são equivalentes:

(i) W é um q-design;

(ii) $\sum_{x \in W} R_{k}^{m}(x \cdot y)=0, y \in S^{m-1}, k=1,2, \ldots, q$;

(iii) Vale a fórmula

$$
\frac{1}{|W|} \sum_{x \in W}(x \cdot y)^{k}=\alpha_{k}, \quad y \in S^{m-1}, \quad k=1,2, \ldots, q,
$$

onde

$$
\alpha_{k}= \begin{cases}0, & k \text { impar } \\ \frac{\sigma_{m-2}}{\sigma_{m-1}} \int_{-1}^{1} t^{k}\left(1-t^{2}\right)^{(m-3) / 2} d t, & k \text { par }\end{cases}
$$

Prova: Assuma que $W$ é um $q$-design. Fixe $y \in S^{m-1}$ e $k \in\{1,2, \ldots, q\}$. Como a função $x \in \mathbb{R} \rightarrow R_{k}^{m}(x \cdot y)$ é um elemento de $\mathcal{P}_{q}$ segue que

$$
\frac{1}{|W|} \sum_{x \in W} R_{k}^{m}(x \cdot y)=\frac{1}{\sigma_{m-1}} \int_{S^{m-1}} R_{k}^{m}(x \cdot y) d \sigma(x) .
$$

Usando o Teorema 1.3.1 obtemos

$$
\begin{aligned}
\frac{1}{|W|} \sum_{x \in W} R_{k}^{m}(x \cdot y) & =\frac{1}{N_{k}} \sum_{j=1}^{N_{k}} \int_{S^{m-1}} Y_{j}^{k}(x) Y_{j}^{k}(y) d \sigma(x) \\
& =\frac{1}{N_{k}} \sum_{j=1}^{N_{k}} Y_{j}^{k}(y) \int_{S^{m-1}} Y_{j}^{k}(x) d \sigma(x) .
\end{aligned}
$$

Pelo Corolário 1.1.1 concluímos que

$$
\frac{1}{|W|} \sum_{x \in W} R_{k}^{m}(x \cdot y)=0 .
$$

Isto mostra que $(i)$ implica $(i i)$.

A seguir, assuma que $(i i)$ vale. Fixemos $y \in S^{m-1}$ e $k \in\{1,2, \ldots, q\}$. Se $k$ é ímpar, digamos, $k=2 s+1$, usamos o Lema 1.6.2 para escrever

$$
\sum_{x \in W}(x \cdot y)^{2 s+1}=\sum_{j=0}^{s} c_{2 j+1} \sum_{x \in W} R_{2 j+1}^{m}(x \cdot y) .
$$


A hipótese (ii) implica diretamente que

$$
\sum_{x \in W}(x \cdot y)^{2 s+1}=0
$$

Se $k$ é par, digamos, $k=2 s$, um procedimento análogo nos leva a

$$
\sum_{x \in W}(x \cdot y)^{2 s}=\sum_{j=0}^{s} c_{2 j} \sum_{x \in W} R_{2 j}^{m}(x \cdot y)=c_{0} \sum_{x \in W} 1=c_{0}|W|
$$

Integrando a igualdade do Lema 1.6.2, obtemos

$$
\int_{-1}^{1} t^{2 s}\left(1-t^{2}\right)^{(m-3) / 2} d t=c_{0} \int_{-1}^{1}\left(1-t^{2}\right)^{(m-3) / 2} d t+\sum_{j=1}^{s} c_{2 j} \int_{-1}^{1} R_{2 j}^{m}(t)\left(1-t^{2}\right)^{(m-3) / 2} d t .
$$

Segue do Corolário 1.1.1 e do Lema 1.6.3 que

$$
\int_{-1}^{1} t^{2 s}\left(1-t^{2}\right)^{(m-3) / 2} d t=c_{0} \frac{\sigma_{m-1}}{\sigma_{m-2}}
$$

Portanto,

$$
\frac{1}{|W|} \sum_{x \in W}(x \cdot y)^{2 s}=\frac{\sigma_{m-2}}{\sigma_{m-1}} \int_{-1}^{1} t^{2 s}\left(1-t^{2}\right)^{\frac{m-3}{2}} d t
$$

Sendo assim, vamos assumir que (iii) vale e concluir que $W$ é um $q$-design. Inicialmente vamos verificar que (2.1) vale para as funções da forma

$$
f(x)=R_{k}^{m}(x \cdot y), \quad x \in S^{m-1}, \quad k=0,1, \ldots, q
$$

Escrevendo

$$
R_{k}^{m}(t)=\sum_{i=0}^{\lfloor k / 2\rfloor} a_{k-2 i} t^{k-2 i}
$$

obtemos

$$
\frac{1}{|W|} \sum_{x \in W} R_{k}^{m}(x \cdot y)=\sum_{i=0}^{\lfloor k / 2\rfloor} a_{k-2 i} \frac{1}{|W|} \sum_{x \in W}(x \cdot y)^{k-2 i} .
$$

Se $k$ é ímpar, cada somando acima anula-se. Caso contrário, primeiro observe que a condição (iii) implica que

$$
\frac{1}{|W|} \sum_{x \in W}(x \cdot y)^{k-2 i}=\frac{\sigma_{m-2}}{\sigma_{m-1}} \int_{-1}^{1} t^{k-2 i}\left(1-t^{2}\right)^{(m-3) / 2} d t
$$

ou seja,

$$
\frac{1}{|W|} \sum_{x \in W} R_{k}^{m}(x \cdot y)=\sum_{i=0}^{\lfloor k / 2\rfloor} a_{k-2 i} \alpha_{k-2 i}
$$


Por outro lado, usando (1.14) e o Teorema 1.5.1 vemos que

$$
\frac{1}{\sigma_{m-1}} \int_{S^{m-1}} R_{k}^{m}(x \cdot y) d \sigma(y)=\sum_{i=0}^{\lfloor k / 2\rfloor} a_{k-2 i} \alpha_{k-2 i},
$$

no caso em que $k$ é par, enquanto que essa mesma integral anula-se nos demais casos, devido à ortogonalidade dos harmônicos esféricos. Como $\mathcal{A}_{k}$ pode ser gerado por um conjunto da forma

$$
\left\{R_{k}^{m}\left(x \cdot x_{j}\right): j=1,2, \ldots, N_{k}\right\},
$$

onde $\left\{x_{j}: j=1,2, \ldots, N_{k}\right\}$ é um sistema fundamental de $S^{m-1}$ (Corolário 1.4.1), concluimos que a fórmula (2.1) vale para os elementos de $\cup_{k=0}^{q} \mathcal{A}_{k}$. Pela Observação 2.1.1, a fórmula vale para elementos de $\mathcal{P}_{q}$.

Observação 2.1.2. A Fórmula de Funk-Hecke gera a seguinte formulação alternativa para os coeficientes do item (iii)

$$
\alpha_{k}= \begin{cases}0, & k \text { impar } \\ \frac{1}{\sigma_{m-1}} \int_{S^{m-1}}(a \cdot x)^{k} d \sigma(x), & k \text { par }\end{cases}
$$

onde $a$ é um ponto arbitrário de $S^{m-1}$.

Exemplo 2.1.3. ( $m=2) O$ conjunto $W=\{(1,0),(0,1),(-1,0),(0,-1)\}$ (vértices de um quadrado) é um 3-design.

De fato, seja $y=\left(y_{1}, y_{2}\right) \in S^{1}$. Se $n=1$, a soma em (iii) toma a forma

$$
y_{1}-y_{1}+y_{2}-y_{2}=0 \text {. }
$$

Se $n=2$, ela reduz-se a

$$
\left.y_{1}^{2}+\left(-y_{1}\right)^{2}+y_{2}^{2}+\left(-y_{2}\right)^{2}\right)=2\left(y_{1}^{2}+y_{2}^{2}\right)=2|y|^{2} .
$$

Por outro lado, tomando-se $a=(1,0)$ em $(2.1 .2)$ e escrevendo $y=(\cos t, \sin t)$, obtemos

$$
\alpha_{2}=\frac{1}{2 \pi} \int_{S^{1}}\left((1,0) \cdot\left(y_{1}, y_{2}\right)\right)^{2} d \sigma(y)=\frac{1}{2 \pi} \int_{0}^{2 \pi} \cos ^{2} t d t=\frac{1}{2} .
$$

Finalmente, se $n=3$,

$$
y_{1}^{3}+\left(-y_{1}\right)^{3}+y_{2}^{3}+\left(-y_{2}\right)^{3}=0
$$

Logo, $W$ é um 3-design devido ao Teorema 2.1.1-(iii). 
Exemplo 2.1.4. ( $m=3)$ O conjunto

$$
W=\{(1,0,0),(0,1,0),(0,0,1),(-1,0,0),(0,-1,0),(0,0,-1)\}
$$

(vértices de um octaedro) é um 3-design.

Vamos verificar isso usando o Teorema 2.1.1-(ii). Para tanto, lembramos que os polinômios de Legendre neste caso são dados por

$$
R_{1}^{m}(t)=t, \quad R_{2}^{m}(t)=\frac{3}{2} t^{2}-\frac{1}{2}, \quad R_{3}^{m}(t)=\frac{5}{2} t^{3}-\frac{3}{2} t
$$

Fixemos $y=\left(y_{1}, y_{2}, y_{3}\right) \in S^{2}$. Se $n=1$,

$$
\sum_{x \in W} R_{1}^{3}(x \cdot y)=y_{1}+y_{2}+y_{3}-y_{1}-y_{2}-y_{3}=0 .
$$

Se $n=2$,

$$
\sum_{x \in W} R_{2}^{3}(x \cdot y)=\frac{1}{2}\left(6 y_{1}^{2}+6 y_{2}^{2}+6 y_{3}^{2}-6\right)=3|y|^{2}-3=0 .
$$

Finalmente, se $n=3$,

$$
\sum_{x \in W} R_{3}^{3}(x \cdot y)=\frac{1}{2}\left(5 y_{1}^{3}+5 y_{2}^{3}+5 y_{3}^{3}-5 y_{1}^{3}-5 y_{2}^{3}-5 y_{2}^{3}-3 y_{1}-3 y_{2}-3 y_{3}+3 y_{1}+3 y_{2}+3 y_{3}\right)=0 .
$$

Exemplo 2.1.5. O conjunto

$$
W=\left\{\frac{1}{\sqrt{m}}( \pm 1, \pm 1, \ldots, \pm 1)\right\}
$$

$\left(2^{m}\right.$ vértices do $m$-cubo $\left.[-1,1]^{m}\right)$ é um 3-design em $S^{m-1}$.

Vamos verificar isso usando o Teorema 2.1.1-(ii). Como $W=-W$ e os polinômios de Legendre de grau ímpar são funções ímpares segue que

$$
\sum_{x \in W} R_{n}^{m}(x \cdot y)=0, \quad y \in S^{m-1}, \quad n \text { ímpar. }
$$

Resta verificar a condição no caso em que $n=2$. Como

$$
\sum_{x \in W}(x \cdot y)^{2}=\frac{1}{m}\left( \pm y_{1}, \pm y_{2}, \ldots, \pm y_{m}\right)^{2}=\frac{2}{m}\left(y_{1}^{2}+y_{2}^{2}+\cdots+y_{m}^{2}\right)=\frac{2}{m}
$$

temos que

$$
\sum_{x \in W} R_{2}^{m}(x \cdot y)=\frac{1}{m-1}\left(\sum_{x \in W}\left(m(x \cdot y)^{2}-1\right)\right)=\frac{1}{m-1}\left(m \frac{2}{m}-2\right)=0 .
$$




\subsection{Designs esféricos e invariância}

Os teoremas abaixo fornecem algumas equivalências adicionais para o conceito de design esférico. A invariância por elementos de $\mathcal{O}(m)$ é o conceito adicional que introduzimos no capítulo 1.

Teorema 2.2.1. Seja $W$ um subconjunto finito e não-vazio de $S^{m-1}$. Então as seguintes afirmações são equivalentes:

(i) W é um q-design;

(ii) Vale a fórmula

$$
\frac{1}{|W|} \sum_{x \in W} p(\rho(x))=\frac{1}{\sigma_{m-1}} \int_{S^{m-1}} p(x) d \sigma(x), p \in \mathcal{H}_{k}, k=0,1, \ldots, q, \rho \in \mathcal{O}_{m} .
$$

(iii) $\sum_{x \in W} h(x)=0, h \in \mathcal{A}_{k}, k=1,2, \ldots, q$;

(iv) $\sum_{x \in W} p(\rho(x))=\sum_{x \in W} p(x), p \in \mathcal{H}_{k}, k=0,1, \ldots, q, \rho \in \mathcal{O}_{m}$.

Prova: Sejam $k \in\{0,1, \ldots, q\}, p \in \mathcal{H}_{k}$ e $\rho \in \mathcal{O}_{m}$. Se $(i)$ vale então a invariância da medida $\sigma$ em relação a elementos de $\mathcal{O}_{m}$ implica que

$$
\sum_{x \in W} p(\rho(x))=\frac{1}{\sigma_{m-1}} \int_{S^{m-1}} p(\rho(x)) d \sigma(x)=\frac{1}{\sigma_{m-1}} \int_{S^{m-1}} p(x) d \sigma\left(\rho^{*}(x)\right)
$$

uma vez que $p \circ \rho \in \mathcal{P}_{q}$. Logo, $(i)$ implica (ii). Para verificar que (ii) implica (iii) basta usar $\rho=I_{m}$ em $($ ii $)$, notar que $\mathcal{A}_{k} \subset \mathcal{H}_{k}$ e então usar o Corolário 1.1.1. Assuma agora que (iii) vale. Sejam $k \in\{1,2, \ldots, q\}, p \in \mathcal{H}_{k}$ e $\rho \in \mathcal{O}_{m}$. Pelo Teorema 1.1.3 podemos escrever

$$
p(x)=\sum_{j=0}^{\lfloor k / 2\rfloor} h_{k-2 j}(x), \quad h_{k-2 j} \in \mathcal{A}_{k-2 j}, \quad j=0,1, \ldots,\lfloor k / 2\rfloor .
$$

Concluiremos que $(i v)$ vale em duas etapas. Se $k$ é ímpar, $k-2 j \geq 1, j=0,1, \ldots,\lfloor k / 2\rfloor$ e, conseqüentemente,

$$
\sum_{x \in W} p(x)=\sum_{j=0}^{\lfloor k / 2\rfloor} \sum_{x \in W} h_{k-2 j}(x)=0
$$

Por outro lado, como $h_{k-2 j} \circ \rho \in \mathcal{A}_{k}, j=0,1, \ldots,\lfloor k / 2\rfloor$, um procedimento similar revela que $\sum_{x \in W} p(\rho(x))=0$. Se $k$ é par, procedemos da mesma forma para encontrar

$$
\sum_{x \in W} p(x)=|W|=\sum_{x \in W} p(\rho(x))
$$


Em qualquer caso, concluímos que

$$
\sum_{x \in W} p(x)=\sum_{x \in W} p(\rho(x))
$$

Para finalizarmos a prova, mostraremos que (iv) implica na condição (iii) do teorema anterior. Seja $k \in\{0,1, \ldots, q\}$. Para cada $y \in S^{m-1}$, tomemos $\rho_{y} \in \mathcal{O}_{m}$ de modo que as coordenadas de $y$ em relação à base canônica de $\mathbb{R}^{m}$ sejam as entradas na primeira linha da matriz de $\rho_{y}$ em relação à mesma base. Então

$$
\sum_{x \in W}(x \cdot y)^{k}=\sum_{x \in W} p\left(\rho_{y}(x)\right)
$$

onde $p(x)=x_{1}^{k}\left(x_{1}=\right.$ primeira componente de $\left.x\right)$. Se $(i v)$ vale, concluímos que

$$
\sum_{x \in W}(x \cdot y)^{k}=\sum_{x \in W} p(x)
$$

Segue que a função $y \in S^{m-1} \rightarrow \sum_{x \in W}(x \cdot y)^{k}$ é uma constante independente de $y$. Seja $\beta$ tal constante. Por integração temos que

$$
\beta=\frac{1}{\sigma_{m-1}} \int_{S^{m-1}} \beta d \sigma(y)=\frac{1}{\sigma_{m-1}} \int_{S^{m-1}} \sum_{x \in W}(x \cdot y)^{k} d \sigma(y)=\sum_{x \in W} \frac{1}{\sigma_{m-1}} \int_{S^{m-1}}(x \cdot y)^{k} d \sigma(y) .
$$

Pela Observação 2.1.2 concluímos que

$$
\beta=|W| \alpha_{k}
$$

como desejado.

Como conseqüencia temos o seguinte resultado original.

Teorema 2.2.2. Seja $W$ um subconjunto finito e não-vazio de $S^{m-1}$. Então as seguintes afirmações são equivalentes:

(i) W é um q-design;

(ii) $\sum_{x \in W} R_{k}^{m}(\rho(x) \cdot y)=\sum_{x \in W} R_{k}^{m}(x \cdot y), k=1,2, \ldots, q, y \in S^{m-1}, \rho \in \mathcal{O}_{m}$.

Prova: Como cada função $x \in S^{m-1} \rightarrow R_{k}^{m}(x \cdot y), y \in S^{m-1}$ é um elemento de $\mathcal{H}_{k}$, uma implicação segue diretamente do teorema anterior. Para a outra, basta verificar que a condição (ii) implica na condição $(i v)$ do Teorema 2.2.1. Para tanto, em vista do Teorema 1.1.3, basta verificar a última condição acima no caso em que $h \in \mathcal{A}_{k}, k=0,1, \ldots, q$. 
Como o caso $k=0$ é trivial, fixe $k \in\{1,2, \ldots, q\}$ e seja $h \in \mathcal{A}_{k}$. Notando que $\alpha_{k}\left(R_{k}^{m}\right)>0$, a Fórmula de Funk-Hecke produz a igualdade

$$
\begin{aligned}
\sum_{x \in W} h(x) & =\frac{1}{\alpha_{k}\left(R_{k}^{m}\right)} \sum_{x \in W} \int_{S^{m-1}} R_{k}^{m}(x \cdot y) h(y) d \sigma(y) \\
& =\frac{1}{\alpha_{k}\left(R_{k}^{m}\right)} \int_{S^{m-1}}\left(\sum_{x \in W} R_{k}^{m}(x \cdot y)\right) h(y) d \sigma(y) .
\end{aligned}
$$

Se $(i i)$ vale, a igualdade acima implica que

$$
\sum_{x \in W} h(x)=\sum_{x \in W} h(\rho(x)), \quad \rho \in \mathcal{O}(m)
$$

Isto completa a prova.

\subsection{Designs esféricos via formas quadráticas}

Apresentamos aqui equivalências para o conceito de designs esféricos que de certa forma simplificam as demais já apresentadas. Os argumentos envolvem a não-negatividade definida de certas formas quadráticas. Como agravante, as somas baseadas em $W$ passam a ser somas duplas.

Teorema 2.3.1. Seja $W$ um subconjunto finito e não-vazio de $S^{m-1}$. Então as seguintes afirmações são equivalentes:

(i) W é um q-design;

(ii) $\sum_{x \in W} \sum_{y \in W}(x \cdot y)^{k}=\alpha_{k}|W|^{2}, k=q-1, q$;

(iii) $\sum_{x \in W} \sum_{y \in W} R_{k}^{m}(x \cdot y)=0, k=1,2, \ldots, q$;

(iv) $\sum_{x \in W} \sum_{y \in W} \mathcal{G}_{q}(x \cdot y)=|W|^{2}$.

Prova: Se $(i)$ vale, usamos a Condição (iii) do Teorema 2.1.1 para obter

$$
\sum_{x \in W} \sum_{y \in W}(x \cdot y)^{k}=|W| \sum_{y \in W} \alpha_{k}=\alpha_{k}|W|^{2}, \quad k=1,2, \ldots, q .
$$

Em particular, (ii) vale.

Suponha agora que $(i i)$ vale. Usando o Lema 1.6.2, vemos que

$$
\begin{aligned}
\alpha_{k} & =\frac{1}{|W|^{2}} \sum_{x \in W} \sum_{y \in W}(x \cdot y)^{k} \\
& =\frac{1}{|W|^{2}} \sum_{j=0}^{\lfloor k / 2\rfloor} c_{k-2 j} \sum_{x \in W} \sum_{y \in W} R_{k-2 j}^{m}(x \cdot y) .
\end{aligned}
$$


Assuma agora que $k \in\{q-1, q\}$. Se $k$ é ímpar, $\alpha_{k}=0$ e usando a positividade dos coeficientes oriundos do Lema 1.6.2, podemos concluir que

$$
\sum_{x \in W} \sum_{y \in W} R_{k-2 j}^{m}(x \cdot y)=0, \quad j=0,1, \ldots,\lfloor k / 2\rfloor .
$$

Se $k$ é par, após notarmos que $\alpha_{k}=c_{0}$, usamos o mesmo argumento para obter a mesma conclusão para $j=1,2, \ldots,\lfloor k / 2\rfloor$. A condição (iii) segue.

Que (iii) implica $(i v)$, segue diretamente da definição de $\mathcal{G}_{q}$.

Para concluir a prova, mostraremos que $(i v)$ implica $(i)$. Usando a Fórmula da Adição temos que

$$
\begin{aligned}
\sum_{x \in W} \sum_{y \in W} \mathcal{G}_{q}(x \cdot y) & =N_{0}|W|^{2}+\sum_{k=1}^{q} N_{k} \sum_{x \in W} \sum_{y \in W} R_{k}^{m}(x \cdot y) \\
& =|W|^{2}+\sum_{k=1}^{q} N_{k} \frac{\sigma_{m-1}}{N_{k}} \sum_{j=1}^{N_{k}} \sum_{x \in W} \sum_{y \in W} Y_{j}^{k}(x) Y_{j}^{k}(y) \\
& =|W|^{2}+\sigma_{m-1} \sum_{k=1}^{q} \sum_{j=1}^{N_{k}}\left(\sum_{x \in W} Y_{j}^{k}(x)\right)^{2}
\end{aligned}
$$

Logo, (iv) reduz a igualdade acima a

$$
\sum_{k=1}^{q} \sum_{j=1}^{N_{k}}\left(\sum_{x \in W} Y_{j}^{k}(x)\right)^{2}=0
$$

ou seja, concluímos que

$$
\sum_{x \in W} h(x)=0, \quad h \in \mathcal{A}_{k}, \quad k=1,2, \ldots, q .
$$

Todavia, devido à ortogonalidade dos harmônicos esféricos, temos que

$$
\int_{S^{m-1}} h(x) d \sigma(x)=0, \quad h \in \mathcal{A}_{k}, \quad k=1,2, \ldots, q .
$$

Segue que

$$
\frac{1}{\sigma_{m-1}} \int_{S^{m-1}} h(x) d \sigma(x)=\frac{1}{|W|} \sum_{x \in W} h(x), \quad h \in \mathcal{A}_{k}, \quad k=1,2, \ldots, q .
$$

Como essa igualdade é obviamente válida para elementos de $\mathcal{A}_{0}$, então ela é válida para todos os elementos de $\mathcal{P}_{q}$.

O teorema a seguir nos fornece uma maneira mais simples para verificar se um determinado conjunto é um q-design, do que aquela apresentada em alguns itens do Teorema 2.2.1. 
Teorema 2.3.2. Seja $W$ um subconjunto finito e não-vazio de $S^{m-1}$. Então as seguintes afirmações são equivalentes:

(i) W é um q-design;

(ii) Vale a fórmula

$$
|W|^{-1} \sum_{x \in W} p(x)=\sigma_{m-1}^{-1} \int_{S^{m-1}} p(y) d \sigma(y), \quad p \in \mathcal{H}_{l}, \quad l=q-1, q
$$

(iii) $\sum_{x \in W} p(x)=\sum_{x \in W} p(\rho(x)), \rho \in \mathcal{O}_{m}, p \in \mathcal{H}_{l}, \quad l=q-1, q$.

Prova: A Definição 2.1.1 mostra que $(i)$ implica $(i i)$. Se (ii) vale, podemos usar a invariância da medida $\sigma$ por elementos de $\mathcal{O}_{m}$ para concluir que, se $p \in \mathcal{H}_{l}, l=q-1, q$, então

$$
\begin{aligned}
\sum_{x \in W} p(\rho(x)) & =\frac{|W|}{\sigma_{m-1}} \int_{S^{m-1}} p(\rho(y)) d \sigma(y) \\
& =\frac{|W|}{\sigma_{m-1}} \int_{S^{m-1}} p(y) d \sigma\left(\rho^{*}(y)\right)=\sum_{x \in W} p(x), \quad l=q-1, q
\end{aligned}
$$

Logo, (iii) vale. Finalmente, vamos mostrar que (iii) implica no item (ii) do Teorema 2.3.1. Por um raciocínio análogo ao da demonstração do Teorema 2.2.1 temos que

$$
\sum_{x \in W}(x \cdot y)^{l}=\beta, \quad l=q-1, q
$$

Portanto, ao somar ambos os lados da igualdade acima em $y \in W$, e utilizar (2.4) obtemos

$$
\sum_{y \in W} \sum_{x \in W}(x \cdot y)^{l}=|W|^{2} \alpha_{l}, \quad l=q-1, q
$$

Isso conclui o teorema.

O próximo teorema nos dá uma caracterização de design esférico utilizando o produto interno definido em (1.1). Lembramos a notação introduzida antes da Proposição 1.1.3, aqui usada para os espaços $\mathcal{A}_{k}$ e $\mathcal{A}_{l}$.

Teorema 2.3.3. Seja $W$ um subconjunto finito e não-vazio de $S^{m-1}$. Então as seguintes afirmações são equivalentes:

(i) W é um q-design;

(ii) $|W|\langle f, g\rangle=\sigma_{m-1} \sum_{x \in W} f(x) g(x), f, g, f g \in \mathcal{P}_{q}$;

(iii) Vale a fórmula $\sigma_{m-1} \sum_{x \in W} Y_{i}^{k}(x) Y_{j}^{n}(x)=|W| \delta_{k, n} \delta_{i, j}$, quando $k=n=\lfloor q / 2\rfloor e$ $k=\lfloor q / 2\rfloor=n+(-1)^{q}$. 
Prova: A Definição 2.1.1 mostra que $(i)$ implica (ii). Por outro lado, quando $k=n=$ $\lfloor q / 2\rfloor$ ou $k=\lfloor q / 2\rfloor=n+(-1)^{q}$, o produto $Y_{i}^{k} Y_{j}^{n}$ pertence a $\mathcal{P}_{q}$. Logo, (ii) implica (iii). Para finalizar, mostraremos que (iii) implica na condição (iii) do Teorema 2.3.1. Para isso consideraremos dois casos.

Caso 1: $k=n=\lfloor q / 2\rfloor$. Neste caso temos que $\sigma_{m-1} \sum_{x \in W} Y_{i}^{k}(x) Y_{j}^{k}(x)=|W| \delta_{i, j}$. Conseqüentemente,

$$
\sum_{i=1}^{N_{k}} \sum_{j=1}^{N_{k}}\left(\sum_{x \in W} Y_{i}^{k}(x) Y_{j}^{k}(x)\right)^{2}=\sum_{i=1}^{N_{k}} \sum_{j=1}^{N_{k}}\left(\frac{|W|}{\sigma_{m-1}} \delta_{i, j}\right)^{2}=\frac{|W|^{2}}{\sigma_{m-1}^{2}} N_{k} .
$$

Logo,

$$
\begin{aligned}
\sum_{x \in W} \sum_{y \in W} R_{k}^{m}(x \cdot y) R_{k}^{m}(x \cdot y) & =\left(\frac{\sigma_{m-1}}{N_{k}}\right)^{2} \sum_{i=1}^{N_{k}} \sum_{j=1}^{N_{k}} \sum_{x \in W} \sum_{y \in W} Y_{i}^{k}(x) Y_{i}^{k}(y) Y_{j}^{k}(x) Y_{j}^{k}(y) \\
& =\left(\frac{\sigma_{m-1}}{N_{k}}\right)^{2} \sum_{i=1}^{N_{k}} \sum_{j=1}^{N_{k}}\left(\sum_{x \in W} Y_{i}^{k}(x) Y_{j}^{k}(x)\right)\left(\sum_{y \in W} Y_{i}^{k}(y) Y_{j}^{k}(y)\right) \\
& =\frac{|W|^{2}}{N_{k}} .
\end{aligned}
$$

Pela Fórmula de Dougall concluimos que

$$
\begin{aligned}
\frac{|W|^{2}}{N_{k}} & =\sum_{x \in W} \sum_{y \in W} R_{k}^{m}(x \cdot y) R_{k}^{m}(x \cdot y) \\
& =\sum_{x \in W} \sum_{y \in W} \sum_{j=0}^{k} \alpha_{j}^{k, k} R_{2 k-2 j}^{m}(x \cdot y) \\
& =\sum_{x \in W} \sum_{y \in W} \alpha_{k}^{k, k} R_{0}^{m}(x \cdot y)+\sum_{x \in W} \sum_{y \in W} \sum_{j=0}^{k-1} \alpha_{j}^{k, k} R_{2 k-2 j}^{m}(x \cdot y) \\
& =|W|^{2} \alpha_{k}^{k, k}+\sum_{x \in W} \sum_{y \in W} \sum_{j=0}^{k-1} \alpha_{j}^{k, k} R_{2 k-2 j}^{m}(x \cdot y) .
\end{aligned}
$$

Como os coeficientes $\alpha_{j}^{k, k}$ são positivos, a Proposição 1.6.1 e o Corolário 1.3.1 implicam que

$$
\sum_{x \in W} \sum_{y \in W} R_{\mu}^{m}(x \cdot y)=0, \quad \mu=2,4, \ldots, q .
$$

Caso 2: $k=\lfloor q / 2\rfloor=n+(-1)^{q}$. Aqui temos que $\sum_{x \in W} Y_{i}^{k}(x) Y_{j}^{n}(x)=0$, pois $k \neq n$. Empregando os mesmos argumentos usados acima chegamos à seguinte igualdade

$$
0=\sum_{x \in W} \sum_{y \in W} R_{k}^{m}(x \cdot y) R_{n}^{m}(x \cdot y)=\sum_{x \in W} \sum_{y \in W} \sum_{j=0}^{k \wedge n} \alpha_{j}^{k, n} R_{2 k-2 j}^{m}(x \cdot y) .
$$


Se $q$ é par, temos que $k=q / 2$ e $n=q / 2-1$. Logo,

$$
\sum_{x \in W} \sum_{y \in W} \sum_{j=0}^{q / 2-1} \alpha_{j}^{k, q / 2-1} R_{q-1-2 j}^{m}(x \cdot y)=0
$$

ou seja,

$$
\sum_{x \in W} \sum_{y \in W} R_{\mu}^{m}(x \cdot y)=0, \quad \mu=1,3, \ldots, q-1
$$

Se $q$ é ímpar, temos uma conclusão similar, qual seja, para $\mu=1,3, \ldots, q$. Assim,

$$
\sum_{x \in W} \sum_{y \in W} R_{\mu}^{m}(x \cdot y)=0, \quad \mu=1,2, \ldots, q
$$

em qualquer caso.

\subsection{Resultados Adicionais}

Esta seção contém uma equivalência adicional para o conceito de design esférico. A condição suficiente apresentada no Teorema 2.4.2, é fundamental na prova dos resultados do Capítulo 3. A referência [9] afirma que a condição é também necessária mas não conseguimos ratificar essa informação.

Teorema 2.4.1. Seja $W$ um subconjunto finito e não-vazio de $S^{m-1}$. Então as seguintes afirmações são equivalentes:

(i) W é um q-design;

(ii) $\delta_{k, l}|W| h(y)=\sum_{x \in W} N_{l} h(x) R_{l}^{m}(x \cdot y), 0 \leq k+l \leq q, h \in \mathcal{A}_{k}, y \in S^{m-1}$.

Prova: Assumindo $(i)$, podemos usar o Teorema 2.3.3-(ii), juntamente com o Teorema 
1.3.1. Logo, se $k, l, h$ e $y$ são como em $(i i)$ temos que

$$
\begin{aligned}
\sum_{x \in W} N_{l} Y_{i}^{k}(x) R_{l}^{m}(x \cdot y) & =\sigma_{m-1} \sum_{x \in W} Y_{i}^{k}(x) \sum_{j=1}^{N_{l}} Y_{j}^{l}(x) Y_{j}^{l}(y) \\
& =\sigma_{m-1} \sum_{j=1}^{N_{l}}\left(\sum_{x \in W} Y_{i}^{k}(x) Y_{j}^{l}(x)\right) Y_{j}^{l}(y) \\
& =|W| \sum_{j=1}^{N_{l}} \delta_{k, l} \delta_{i, j} Y_{j}^{l}(y) \\
& =|W| \delta_{k, l} Y_{i}^{l}(y) \\
& =|W| \delta_{k, l} Y_{i}^{k}(y), \quad i=1,2, \ldots, N_{k} .
\end{aligned}
$$

Quanto à recíproca, notemos inicialmente que a fórmula em (ii) pode ser reescrita na forma

$$
\sigma_{m-1} \sum_{j=1}^{N_{l}}\left(\sum_{x \in W} Y_{\mu}^{k}(x) Y_{j}^{l}(x)\right) Y_{j}^{l}=\delta_{\mu, j}|W| Y_{\mu}^{k}
$$

quando usamos $h=Y_{\mu}^{k}$. Substituindo $k=l=\lfloor q / 2\rfloor$ e $k=\lfloor q / 2\rfloor=l+(-1)^{q}$ e usando o fato de que o conjunto $\left\{Y_{j}^{l}: j=1,2, \ldots, N_{l}\right\}$ é l.i., obtemos imediatamente a condição (iii) do Teorema 2.3.3.

Lembrando o Teorema 2.3.3-(ii), o resultado abaixo estabelece uma espécie de fórmula de reprodução baseada em $W$ para os elementos de $\mathcal{P}_{k}, k=0,1, \ldots,\lfloor q / 2\rfloor$, com núcleo de reprodução $|W|^{-1} \mathcal{G}_{l}$, onde $l$ satisfaz $k \leq l \leq q-k$, pelo menos quando $W$ é um $q$-design.

Teorema 2.4.2. Se $W$ é um q-design e $k$ e l são inteiros tais que $0 \leq k \leq l \leq q-k$, então

$$
|W| f(y)=\sum_{x \in W} f(x) \mathcal{G}_{l}(x \cdot y), \quad f \in \mathcal{P}_{k}, \quad y \in S^{m-1}
$$

Prova: Devido à Proposição 1.1.2 é suficiente demonstrar o teorema no caso em que 
$f=Y_{\mu}^{n}, n \in\{0,1, \ldots, k\}$ e $\mu \in\left\{1,2, \ldots, N_{k}\right\}$. Empregando o Teorema 2.4.1,

$$
\begin{aligned}
\sum_{x \in W} Y_{\mu}^{n}(x) \mathcal{G}_{l}(x \cdot y) & =\sum_{x \in W} \sum_{j=0}^{l} N_{j} Y_{\mu}^{n}(x) R_{j}^{m}(x \cdot y) \\
& =\sum_{j=0}^{l}\left(\sum_{x \in W} N_{j} Y_{\mu}^{n}(x) R_{j}^{m}(x \cdot y)\right) \\
& =|W| \sum_{j=0}^{l} \delta_{n, j} Y_{\mu}^{n}(y) \\
& =|W| Y_{\mu}^{n}(y) .
\end{aligned}
$$

O teorema segue.

\subsection{Uma aplicação}

Nessa seção $m$ será um inteiro $\geq 3$ e $\varepsilon$ denotará um ponto fixo (polo) de $S^{m-1}$. Vamos representar os pontos de $S^{m-1}$ usando projeções esféricas a partir de $\varepsilon$. Precisamente, se $x$ é um ponto de $S^{m-1}$, temos a decomposição

$$
x=t \varepsilon+\sqrt{1-t^{2}} x_{\varepsilon}^{\prime}, \quad t \in[-1,1], \quad x_{\varepsilon}^{\prime} \in S_{\varepsilon}^{m-1} .
$$

O conjunto

$$
S_{\varepsilon}^{m-1}:=\left\{x \in S^{m-1}: x \cdot \varepsilon=0\right\}
$$

é o "equador" de $S^{m-1}$ ortogonal a $\varepsilon$. Este conjunto é uma cópia exata de $S^{m-2}$ mergulhada isométricamente em $S^{m-1}$.

O mergulho isométrico ao qual nos referimos no parágrafo anterior será denotado por $\Psi_{\varepsilon}$

Dado um subconjunto finito e não-vazio $W$ de $S^{m-1}, W_{\varepsilon}^{\prime}$ denotará a projeção de $W$ a partir de $\varepsilon$, ou seja,

$$
W_{\varepsilon}^{\prime}:=\left\{x_{\varepsilon}^{\prime}: x \in W\right\}
$$

Em particular, temos

$$
S_{\varepsilon}^{m-1}=\left\{x_{\varepsilon}^{\prime}: x \in S^{m-1}\right\} .
$$

O teorema abaixo apresenta um método conciso para construir designs esféricos em $S^{m-2}$ a partir de designs esféricos em $S^{m-1}$. 

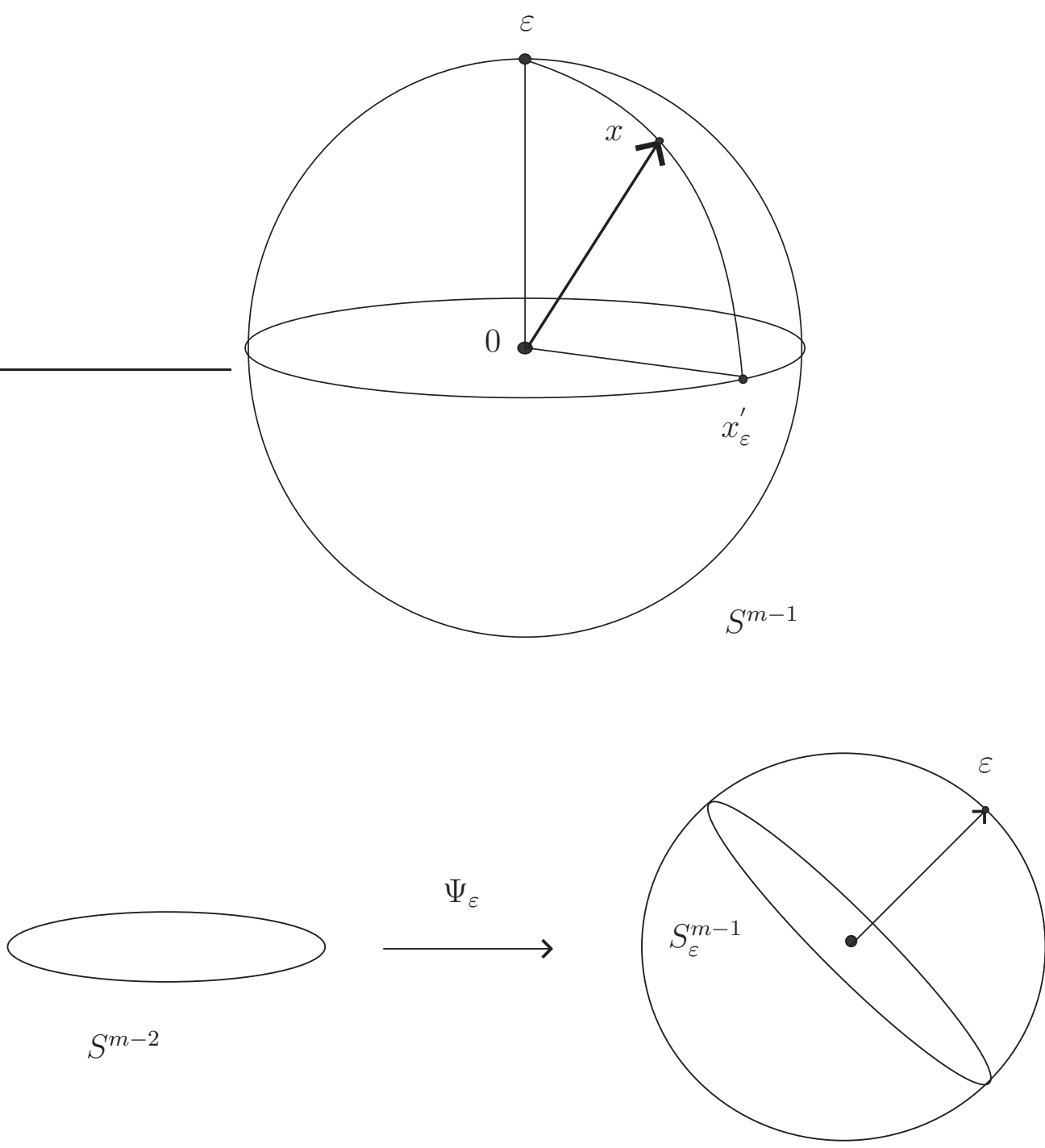

$S^{m-1}$

Teorema 2.5.1. Seja $W$ um conjunto finito e não-vazio de $S^{m-1}$. Assuma que exista $\lambda \in(-1,1) \backslash \cup_{k=0}^{q-1}\left\{t: R_{q-k}^{m+k}(t)=0\right\}$ tal que $W \subset\left\{x \in S^{m-1}: x \cdot \varepsilon=\lambda\right\}$. Se Wé um q-design em $S^{m-1}$, então $\Psi_{\varepsilon}^{-1}\left(W_{\varepsilon}^{\prime}\right)$ é um q-design em $S^{m-2}$.

Prova: É fácil ver que

$$
\sum_{x \in W} \sum_{y \in W} R_{k}^{m}(x \cdot y)=\sum_{x \in W} \sum_{y \in W} R_{k}^{m}\left(\lambda^{2}+\left(1-\lambda^{2}\right) x_{\varepsilon}^{\prime} \cdot y_{\varepsilon}^{\prime}\right), \quad k=0,1, \ldots
$$

Como a aplicação $x \in W \longrightarrow x_{\varepsilon}^{\prime} \in W_{\varepsilon}^{\prime}$ é biunívoca, segue que

$$
\sum_{x \in W} \sum_{y \in W} R_{k}^{m}\left(\lambda^{2}+\left(1-\lambda^{2}\right) x_{\varepsilon}^{\prime} \cdot y_{\varepsilon}^{\prime}\right)=\sum_{x_{\varepsilon}^{\prime} \in W^{\prime}} \sum_{y_{\varepsilon}^{\prime} \in W^{\prime}} R_{k}^{m}\left(\lambda^{2}+\left(1-\lambda^{2}\right) x_{\varepsilon}^{\prime} \cdot y_{\varepsilon}^{\prime}\right)
$$


Se $S_{k}$ denota a última soma dupla acima, decorre do Teorema 1.6 .3 e da fórmula (1.6) que

$$
\begin{aligned}
S_{k} & =\sum_{x_{\varepsilon}^{\prime} \in W_{\varepsilon}^{\prime}} \sum_{y_{\varepsilon}^{\prime} \in W_{\varepsilon}^{\prime}} \sum_{l=0}^{k} \beta_{l, k}^{m}\left(1-\lambda^{2}\right)^{l}\left(R_{k-l}^{m+l}(\lambda)\right)^{2} R_{l}^{m-1}\left(x_{\varepsilon}^{\prime} \cdot y_{\varepsilon}^{\prime}\right) \\
& =\sum_{l=0}^{k} \beta_{l, k}^{m}\left(1-\lambda^{2}\right)^{l}\left(R_{k-l}^{m+l}(\lambda)\right)^{2} \sum_{x_{\varepsilon}^{\prime} \in W_{\varepsilon}^{\prime}} \sum_{y_{\varepsilon}^{\prime} \in W_{\varepsilon}^{\prime}} R_{l}^{m-1}\left(x_{\varepsilon}^{\prime} \cdot y_{\varepsilon}^{\prime}\right),
\end{aligned}
$$

onde

$$
\beta_{l, k}^{m}=\frac{(m-3) ! k !}{(k+m-3) !} \beta_{l, k}, \quad l=0,1, \ldots, k .
$$

Se $W$ é um $q$-design o Teorema 2.3.1-(iii) revela que $S_{k}=0, k=0,1, \ldots, q$. Como os coeficientes $\beta_{l, k}^{m}$ e $\left(1-\lambda^{2}\right)^{l}$ são positivos e $\lambda \notin \cup_{k=0}^{q-1}\left\{t: R_{q-k}^{m+k}(t)=0\right\}$, o Lema 1.3.1 mostra que a condição anterior é equivalente a $\sum_{x_{\varepsilon}^{\prime} \in W_{\varepsilon}^{\prime}} \sum_{y_{\varepsilon}^{\prime} \in W_{\varepsilon}^{\prime}} R_{l}^{m-1}\left(x_{\varepsilon}^{\prime} \cdot y_{\varepsilon}^{\prime}\right)=0, l=0,1, \ldots, q$. Sejam $x^{\prime \prime}$ e $y^{\prime \prime}$ pontos de $S^{m-2}$ tais que $\Psi_{\varepsilon}\left(x^{\prime \prime}\right)=x_{\varepsilon}^{\prime}$ e $\Psi_{\varepsilon}\left(y^{\prime \prime}\right)=y_{\varepsilon}^{\prime}$. Então podemos concluir que

$$
\begin{aligned}
0 & =\sum_{x_{\varepsilon}^{\prime} \in W_{\varepsilon}^{\prime}} \sum_{y_{\varepsilon}^{\prime} \in W_{\varepsilon}^{\prime}} R_{l}^{m-1}\left(x_{\varepsilon}^{\prime} \cdot y_{\varepsilon}^{\prime}\right) \\
& =\sum_{x_{\varepsilon}^{\prime} \in W_{\varepsilon}^{\prime}} \sum_{y_{\varepsilon}^{\prime} \in W_{\varepsilon}^{\prime}} R_{l}^{m-1}\left(\Psi_{\varepsilon}\left(x^{\prime \prime}\right) \cdot \Psi_{\varepsilon}\left(y^{\prime \prime}\right)\right) \\
& =\sum_{x_{\varepsilon}^{\prime} \in W_{\varepsilon}^{\prime}} \sum_{y_{\varepsilon}^{\prime} \in W_{\varepsilon}^{\prime}} R_{l}^{m-1}\left(x^{\prime \prime} \cdot y^{\prime \prime}\right) \\
& =\sum_{x^{\prime \prime} \in \Psi_{\varepsilon}^{-1}\left(W_{\varepsilon}^{\prime}\right)} \sum_{y^{\prime \prime} \in \Psi_{\varepsilon}^{-1}\left(W_{\varepsilon}^{\prime}\right)} R_{l}^{m-1}\left(x^{\prime \prime} \cdot y^{\prime \prime}\right), \quad l=0,1, \ldots, q
\end{aligned}
$$

Pelo Teorema 2.3.1-(iii) novamente, $\Psi_{\varepsilon}^{-1}\left(W_{\varepsilon}^{\prime}\right)$ é um $q$-design em $S^{m-2}$. 


\section{Capítulo 3}

\section{Designs esféricos de cardinalidade mínima}

Assumindo-se que $W$ é um $q$-design, vamos determinar estimativas inferiores e superiores para $|W|$. O problema da existência ou não de $q$-designs com cardinalidade igual ao limitante inferior apresentado não será discutido nesse trabalho, uma vez que essa questão por si só já é muito complicada. Seja $W$ um $q$-design que não é um $(q+1)$-design. Definamos

$$
A_{W}:=\{(x \cdot y): x, y \in W, x \neq y\}
$$

e $s:=\left|A_{W}\right|$.

Teorema 3.0.2. Seja $W$ um q-design esférico. Então

$$
\mathcal{G}_{\lfloor q / 2\rfloor}(1) \leq|W| \leq \mathcal{G}_{s}(1) .
$$

Prova: Consideremos os seguintes conjuntos de polinômios na variável $y$

$$
B_{\lfloor q / 2\rfloor}:=\left\{\mathcal{G}_{\lfloor q / 2\rfloor}(x \cdot y): x \in W\right\}
$$

e

$$
C_{W}:=\left\{\varphi_{x}(y): x \in W\right\},
$$

onde

$$
\varphi_{x}(y):=\prod_{t \in A_{W}} \frac{(x \cdot y)-t}{1-t} .
$$

Vamos aplicar o Teorema 2.4.2 com $l=\lfloor q / 2\rfloor$. Essa escolha de $l$ permite o maior valor possível para $k$ nas condições do teorema (veja discussão no apêndice 1). Pelo teorema 
vemos que $\left[B_{\lfloor q / 2\rfloor}\right]=\mathcal{P}_{\lfloor q / 2\rfloor}$. Como $B_{\lfloor q / 2\rfloor}$ está indexado em $W$ e todo conjunto gerador de um espaço vetorial contém uma base, a primeira desigualdade segue. Como $\varphi_{x}(y)=$ $\delta_{x, y}, x, y \in W$, a igualdade

$$
\sum_{x \in W} c_{x} \varphi_{x}=0, \quad c_{x} \in \mathbb{R}, \quad x \in W
$$

implica necessariamente que $c_{x}=0, x \in W$. Logo, $C_{W}$ é l.i. Como $C_{W}$ também está indexado em $W$ e $C_{W} \subset \mathcal{P}_{s}$, segue que $|W| \leq d_{s}$.

Corolário 3.0.1. Nas condições do teorema anterior vale a desigualdade $\lfloor q / 2\rfloor \leq s$.

O próximo teorema garante a igualdade em (3.1), desde que tenhamos igualdade em apenas um lado. Para os próximos resultados vamos utilizar o Teorema 1.6.2 para expressar

$$
\varphi_{x}(y)=\sum_{\mu=0}^{s} \omega_{\mu} R_{\mu}^{m}(x \cdot y)=\sum_{\mu=0}^{s} \omega_{\mu} \frac{\sigma_{m-1}}{N_{\mu}} \sum_{l=1}^{N_{\mu}} Y_{l}^{\mu}(x) Y_{l}^{\mu}(y),
$$

onde $\omega_{\mu}$ são coeficientes constantes, e o Teorema 1.3.1 para expressar

$$
\mathcal{G}_{s}(x, y)=\sum_{i=0}^{s} N_{i} R_{i}^{m}(x \cdot y)=\sigma_{m-1} \sum_{i=0}^{s} \sum_{j=1}^{N_{i}} Y_{j}^{i}(x) Y_{j}^{i}(y)
$$

Definamos

$$
\Omega_{s}:=\cup_{k=0}^{s}\left\{Y_{1}^{k}, Y_{2}^{k}, \ldots, Y_{N_{k}}^{k}\right\}
$$

Lema 3.0.1. Sejam $\varphi_{x}(z)$ como antes e $Y_{i}^{k} \in \Omega_{s}$. Então

$$
\left\langle\frac{N_{k}}{\omega_{k} \sigma_{m-1}} \varphi_{x}(z), Y_{i}^{k}(z)\right\rangle=Y_{i}^{k}(x)
$$

Prova: Para mostrarmos este fato utilizaremos a fórmula da adição e os resultados acima.

$$
\begin{aligned}
\left\langle\frac{N_{k}}{\omega_{k} \sigma_{m-1}} \varphi_{x}(z), Y_{i}^{k}(z)\right\rangle & =\frac{N_{k}}{\omega_{k} \sigma_{m-1}}\left\langle\sum_{i=0}^{s} \omega_{i} R_{i}^{m}(x \cdot z), Y_{l}^{k}(z)\right\rangle \\
& =\frac{N_{k}}{\omega_{k} \sigma_{m-1}} \sum_{i=0}^{s} \omega_{i} \frac{\sigma_{m-1}}{N_{i}} \sum_{j=0}^{s} Y_{j}^{i}(x)\left\langle Y_{j}^{i}(z), Y_{l}^{k}(z)\right\rangle \\
& =\frac{N_{k}}{\omega_{k} \sigma_{m-1}} \sum_{i=0}^{s} \omega_{i} \frac{\sigma_{m-1}}{N_{i}} \sum_{j=0}^{s} Y_{j}^{i}(x) \delta_{j, l} \delta_{i, k}=Y_{i}^{k}(x),
\end{aligned}
$$

como desejado. 
Lema 3.0.2. Vale a igualdade $\mathcal{G}_{l}(x \cdot x)=\sigma_{m-1}^{-1} \int_{S^{m-1}} \mathcal{G}_{l}^{2}(x \cdot \xi) d \sigma(\xi)$.

Prova: Este resultado segue da Fórmula da adição. De fato,

$$
\begin{aligned}
\int_{S^{m-1}} \mathcal{G}_{l}^{2}(x \cdot y) d \sigma(y) & =\int_{S^{m-1}} \mathcal{G}_{l}(x \cdot y) \mathcal{G}_{l}(x \cdot y) d \sigma(y) \\
& =\sum_{i=0}^{l} \sum_{j=0}^{l} N_{i} N_{j} \int_{S^{m-1}} R_{i}^{m}(x \cdot y) R_{j}^{m}(x \cdot y) d \sigma(y) \\
& =\sum_{i=1}^{l} \sum_{j=1}^{l} \sigma_{m-1}^{2} \sum_{\mu=0}^{N_{i}} \sum_{\nu=0}^{N_{j}} Y_{\mu}^{i}(x) Y_{\nu}^{j}(x) \int_{S^{m-1}} Y_{\mu}^{i}(y) Y_{\nu}^{j}(y) d \sigma(y) \\
& =\sigma_{m-1}^{2} \sum_{i=0}^{l} \sum_{\mu=1}^{N_{i}} Y_{\mu}^{i}(x) Y_{\mu}^{i}(x) \\
& =\sigma_{m-1} \sum_{i=0}^{l} N_{i} R_{i}^{m}(x \cdot x)=\sigma_{m-1} \mathcal{G}_{l}(x \cdot x)
\end{aligned}
$$

como desejado.

Teorema 3.0.3. Seja $W$ um q-design esférico. As seguintes afirmações são eqüivalentes:

(i) $\mathcal{G}_{\lfloor q / 2\rfloor}(1)=|W|$;

(ii) $|W|=\mathcal{G}_{s}(1)$.

Prova: Suponhamos que $(i)$ vale. Para mostrar que $|W|=\mathcal{G}_{s}(1)$ vamos aplicar a Definição 2.1.1 e o lema anterior para estabelecer a seguinte igualdade

$$
\begin{aligned}
\mathcal{G}_{\lfloor q / 2\rfloor}(1) & =\mathcal{G}_{\lfloor q / 2\rfloor}(x \cdot x) \\
& =\sigma_{m-1}^{-1} \int_{S^{m-1}} \mathcal{G}_{\lfloor q / 2\rfloor}^{2}(x \cdot \xi) d \sigma_{m-1}(\xi) \\
& =|W|^{-1} \sum_{y \in W} \mathcal{G}_{\lfloor q / 2\rfloor}^{2}(x \cdot y)=|W|^{-1} \mathcal{G}_{\lfloor q / 2\rfloor}^{2}(1)+\sum_{\substack{y \in W \\
x \neq y}} \mathcal{G}_{\lfloor q / 2\rfloor}^{2}(x \cdot y), \quad x \in S^{m-1} .
\end{aligned}
$$

Segue que

$$
\sum_{y \in W} \mathcal{G}_{\lfloor q / 2\rfloor}^{2}(x \cdot y)=0, \quad x \neq y
$$

Como $s=\left|A_{W}\right|$, temos que a equação $\mathcal{G}_{\lfloor q / 2\rfloor}=0$ possui $s$ raízes distintas. Desta forma $s \leq\lfloor q / 2\rfloor$ e, portanto, $s=\lfloor q / 2\rfloor$. Reciprocamente, suponhamos que $\mathcal{G}_{s}(1)=|W|$. Decorre do Teorema 1.7.1 que 


$$
\left\langle\varphi_{x}(\star), \sigma_{m-1}^{-1} \mathcal{G}_{s}(y, \star)\right\rangle=\varphi_{x}(y)=\delta_{x, y} .
$$

Assim, podemos concluir que $B_{s}$ é l.i. e consequentemente uma base para $\mathcal{P}_{s}$. Tendo em vista que $W$ é um $2\lfloor q / 2\rfloor$-design e $k \leq s$ podemos empregar o Teorema 2.4.2 e concluir que

$$
\sum_{x \in W}\left\langle\frac{N_{k}}{\omega_{k} \sigma_{m-1}} \varphi_{x}(z), Y_{i}^{k}(z)\right\rangle \mathcal{G}_{s}(x \cdot y)=\sum_{x \in W} Y_{i}^{k}(x) \mathcal{G}_{s}(x \cdot y)=|W| Y_{i}^{k}(y) .
$$

Com base nesses cálculos, vale a seguinte igualdade

$$
\begin{aligned}
\delta_{i, j} \delta_{k, l} & =\left\langle Y_{i}^{k}(y), Y_{j}^{l}(y)\right\rangle \\
& =\left\langle\frac{1}{|W|} \sum_{x \in W}\left\langle\frac{N_{k}}{\omega_{k} \sigma_{m-1}} \varphi_{x}(z), Y_{i}^{k}(z)\right\rangle \mathcal{G}_{s}(x \cdot z), Y_{j}^{l}(z)\right\rangle \\
& =\frac{1}{|W|} \sum_{x \in W}\left\langle\frac{N_{k}}{\omega_{k} \sigma_{m-1}} \varphi_{x}(z), Y_{i}^{k}(z)\right\rangle\left\langle\mathcal{G}_{s}(x \cdot z), Y_{j}^{l}(z)\right\rangle \\
& =\frac{\sigma_{m-1}}{|W|} \sum_{x \in W} Y_{i}^{k}(x)\left\langle\sigma_{m-1}^{-1} \mathcal{G}_{s}(x \cdot z), Y_{j}^{l}(z)\right\rangle \\
& =\frac{\sigma_{m-1}}{|W|} \sum_{x \in W} Y_{i}^{k}(x) Y_{j}^{l}(x), \quad l=0,1, \ldots, s .
\end{aligned}
$$

Logo, pelo Teorema 2.3.3 temos que $W$ é um $2 s$-design. Portanto, $s=\lfloor q / 2\rfloor$, o que conclui o teorema.

Resultados similares podem ser estabelecidos no caso em que os pontos do design comparecem aos pares.

Definição 3.0.1. Um design esférico $W$ em $S^{m-1}$ é chamado antipodal quando $W$ é simétrico em relação à origem de $\mathbb{R}^{m}$.

Os conjuntos e funções definidos no início do capítulo devem então ser substituídos por outros convenientes. O conjunto $A_{W}$ deve ser trocado por

$$
A_{W}:=\{x \cdot y: x, y \in W, x \neq \pm y\}
$$

cuja cardinalidade continuamos a denotar por $s$. Escrevendo $X$ para denotar qualquer conjunto de representantes das classes de equivalência da relação sobre $S^{m-1}$ dada por

$$
x \sim y \quad \text { se, e somente se } y= \pm x,
$$


os conjuntos do início da prova do Teorema 3.0.2 devem ser trocados por

$$
B_{s}:=\left\{\mathcal{Q}_{s}(x \cdot y): x \in X \cap W\right\}
$$

e

$$
C_{W}:=\left\{\varphi_{x}(y): x \in X \cap W\right\}
$$

onde

$$
\mathcal{Q}_{s}:=\sum_{i=0}^{\lfloor n / 2\rfloor} N_{n-2 i} R_{s-2 i}^{m} .
$$

Finalmente, o conjunto $\Omega_{s}$ utilizado na prova do Lema 3.0.1 deve ser trocado por $\Omega_{s}^{\prime}$, uma base de $\mathcal{H}_{s}$.

Os seguintes resultados podem então ser reproduzidos.

Teorema 3.0.4. Seja $W$ um q-design antipodal. Então

$$
2 \mathcal{Q}_{\lfloor q / 2\rfloor}(1) \leq|W| \leq 2 \mathcal{Q}_{s}(1)
$$

Teorema 3.0.5. Seja W um q-design esférico antipodal. As seguintes afirmações são equivalentes:

(i) $2 \mathcal{Q}_{\lfloor q / 2\rfloor}(1)=|W|$;

(ii) $|W|=2 \mathcal{Q}_{s}(1)$.

Exemplos concretos de designs esféricos com cardinalidade coincidindo com o limitante inferior apresentado nos resultados acima podem ser encontrados nas seguintes referências: $[8],[10],[5]$ e $[6]$. 


\section{Capítulo 4}

\section{Rotações de Designs esféricos}

Os resultados desse capítulo ainda exploram as idéias utilizadas na Seção 2.5. A idéia aqui é obter designs novos a partir de um dado design, sem mudar a dimensão da esfera e usando rotações variadas. A importância de resultados desse tipo está ligada ao fato de que, apesar de termos várias equivalências para o conceito de design esférico, a ratificação de que um dado conjunto é de fato um design utilizando qualquer uma dessas equivalências não é trivial.

Em todo este capítulo, a menos de especificação em contrário, $\varepsilon$ é um ponto fixado de $S^{m-1}$. Se $W$ é um conjunto finito e não-vazio de $S^{m-1}$, então é possível decompo-lo em níveis ortogonais ao polo $\varepsilon$ da seguinte forma:

$$
W=\dot{\cup}_{i=1}^{n} W_{\lambda_{i}}, \quad W_{\lambda_{i}}=W \cap\left\{x \cdot \varepsilon=\lambda_{i}: x \in S^{m-1}\right\},
$$

onde $\lambda_{i} \in(-1,1), i=1,2, \ldots, n$.

Teorema 4.0.6. Seja $W$ um conjunto finito e não-vazio de $S^{m-1}$ e considere a decomposição em níveis $W=\dot{\cup}_{i=1}^{n} W_{\lambda_{i}}$. Sejam $\rho_{1}, \rho_{2}, \ldots, \rho_{n}$ elementos de $\mathcal{O}_{m}$ com $\rho_{j}(\varepsilon)=$ $\varepsilon, j=1,2, \ldots, n$. Se $W$ é um q-design e cada $W_{\lambda_{i}, \varepsilon}^{\prime}$ é um $q_{i}$-design, $i=1,2, \ldots, n$, então $W_{\rho}:=\cup_{i=1}^{n} \rho_{i}\left(W_{\lambda_{i}}\right)$ é um $\nu$-design, onde $\nu=q \wedge q_{1} \wedge \ldots \wedge q_{n}$.

Prova: Assuma que $W$ é um $q$-design e que cada $W_{\lambda_{i}, \varepsilon}^{\prime}$ é um $q_{i}$-design, $i=1,2, \ldots, n$. Vamos verificar que

$$
\sum_{x \in W_{\rho}} R_{n}^{m}(x \cdot y)=0, \quad y \in S^{m-1}, \quad n=1,2, \ldots, \nu
$$

Se $\rho_{j}=I_{m}, j=1,2, \ldots, n$, então $W_{\rho}=W$. Como $W$ é um $q$-design, o Teorema 2.1.1 
implica que

$$
\sum_{x \in W_{\rho}} R_{n}^{m}(x \cdot y)=0, \quad y \in S^{m-1}, \quad n=1,2, \ldots, q .
$$

Em particular (4.1) segue. No caso geral vamos escrever

$$
\sum_{x \in W_{\rho}} R_{n}^{m}(x \cdot y)=\sum_{i=1}^{n} \sum_{x \in \rho_{i}\left(W_{\lambda_{i}}\right)} R_{n}^{m}(x \cdot y)
$$

e verificar que o lado direito da igualdade acima independe de $\rho_{i}, i=1,2, \ldots, n$, quando $n \in\{1,2, \ldots, \nu\}$. Para tanto, faremos isso para cada somando. Fixemos um tal $i \in$ $\{1,2, \ldots, n\}$. Como cada $\rho_{i}$ preserva produto interno temos que

$$
\sum_{x \in \rho_{i}\left(W_{\lambda_{i}}\right)} R_{n}^{m}(x \cdot y)=\sum_{\rho_{i}^{*}(x) \in W_{\lambda_{i}}} R_{n}^{m}\left(\rho_{i}^{*}(x) \cdot \rho_{i}^{*}(y)\right)=\sum_{z \in W_{\lambda_{i}}} R_{n}^{m}\left(z \cdot \rho_{i}^{*}(y)\right)
$$

Decompondo z e $y$ através da equação (2.5), obtemos

$$
\begin{aligned}
\sum_{x \in \rho_{i}\left(W_{\lambda_{i}}\right)} R_{n}^{m}(x \cdot y) & =\sum_{z \in W_{\lambda_{i}}} R_{n}^{m}\left(\lambda_{i} \xi+\left(1-\lambda_{i}^{2}\right)^{1 / 2}\left(1-\xi^{2}\right)^{1 / 2}\left(\rho_{i}\left(z_{\varepsilon}^{\prime}\right) \cdot y_{\varepsilon}^{\prime}\right)\right) \\
& =\sum_{z_{\varepsilon}^{\prime} \in W_{\lambda_{i}, \varepsilon}^{\prime}} R_{n}^{m}\left(\lambda_{i} \xi+\left(1-\lambda_{i}^{2}\right)^{1 / 2}\left(1-\xi^{2}\right)^{1 / 2}\left(\rho_{i}\left(z_{\varepsilon}^{\prime}\right) \cdot y_{\varepsilon}^{\prime}\right)\right) .
\end{aligned}
$$

A re-indexação no último somatório justifica-se pelo fato da aplicação $z \in W_{\lambda_{i}} \rightarrow z_{\varepsilon}^{\prime}$ ser biunívoca. Como $R_{n}^{m}$ é um polinômio de grau $n$, existem coeficientes $a_{l}\left(\lambda_{i}, \xi\right), l=$ $0,1, \ldots, n$, de modo que

$$
\sum_{x \in \rho_{i}\left(W_{\lambda_{i}}\right)} R_{n}^{m}(x \cdot y)=\sum_{l=0}^{n} a_{l}\left(\lambda_{i}, \xi\right)\left(1-\lambda_{i}^{2}\right)^{l / 2} \sum_{z_{\varepsilon}^{\prime} \in W_{\lambda_{i}, \varepsilon}^{\prime}}\left(z_{\varepsilon}^{\prime} \cdot \rho_{i}^{*}\left(y_{\varepsilon}^{\prime}\right)\right)^{l} .
$$

Como $W_{\lambda_{i}, \varepsilon}^{\prime}$ é um $q_{i}$-design e $n \leq \nu$, a condição $(i i i)$ do Teorema 2.1.1, pode ser usada para deduzirmos que

$$
\begin{aligned}
\sum_{x \in \rho_{i}\left(W_{\lambda_{i}}\right)} R_{n}^{m}(x \cdot y) & =\left|W_{\lambda_{i}, \varepsilon}^{\prime}\right| \sum_{\substack{l=0 \\
l \text { par }}}^{n} a_{l}\left(\lambda_{i}, \xi\right)\left(1-\lambda_{i}^{2}\right)^{l / 2}\left(\frac{1}{\left|W_{\lambda_{i}, \varepsilon}^{\prime}\right|} \sum_{z_{\varepsilon}^{\prime} \in W_{\lambda_{i}, \varepsilon}^{\prime}}\left(z_{\varepsilon}^{\prime} \cdot \rho_{i}^{*}\left(y_{\varepsilon}^{\prime}\right)\right)^{l}\right) \\
& =\left|W_{\lambda_{i}, \varepsilon}^{\prime}\right| \sum_{\substack{l=0 \\
l \text { par }}}^{n} a_{l}\left(\lambda_{i}, \xi\right)\left(1-\lambda_{i}^{2}\right)^{l / 2} \alpha_{l}\left\|\rho_{i}^{*}\left(y_{\varepsilon}^{\prime}\right)\right\|^{l} .
\end{aligned}
$$

Daí,

$$
\sum_{x \in \rho_{i}\left(W_{\lambda_{i}}\right)} R_{n}^{m}(x \cdot y)=\left|W_{\lambda_{i}, \varepsilon}^{\prime}\right| \sum_{\substack{l=0 \\ l \text { par }}}^{n} a_{l}\left(\lambda_{i}, \xi\right)\left(1-\lambda_{i}^{2}\right)^{l / 2} \alpha_{l}\left\|y_{\varepsilon}^{\prime}\right\|^{l},
$$

uma expressão que independe de $\rho_{i}$. Assim, a condição (4.1) vale e o teorema segue do Teorema 2.1.1. 
Corolário 4.0.2. Sejam $W$ um subconjunto finito e não-vazio de $S^{m-1}$ e $\lambda \in(-1,1)$. Seja $\rho \in \mathcal{O}_{m}$ tal que $\rho(\varepsilon)=\varepsilon$. Se $W$ é um q-design e $W_{\lambda, \varepsilon}^{\prime}$ um $q_{1}$-design, então

$$
W_{\rho}=\left(W \backslash W_{\lambda}\right) \cup \rho\left(W_{\lambda}\right)
$$

é um $q \wedge q_{1}$-design.

Prova: É muito parecida com a prova do Teorema 4.0.6. Aqui, a essência da prova consiste em verificar que a soma

$$
\sum_{x \in W_{\rho}} R_{n}^{m}(x \cdot y)=\sum_{x \in W \backslash W_{\lambda}} R_{n}^{m}(x \cdot y)+\sum_{x \in \rho\left(W_{\lambda}\right)} R_{n}^{m}(x \cdot y),
$$

independe de $\rho$ quando $n \in\left\{1,2, \ldots, q \wedge q_{1}\right\}$, o que pode ser feito da mesma forma.

No teorema a seguir denotaremos por $W_{\lambda}^{\prime \prime}$ o subconjunto de $S^{m-2}$ obtido de $W_{\lambda, \varepsilon}^{\prime}$, quando desprezamos as componentes nulas de seus elementos, que correspondem às entradas não nulas de $\varepsilon$.

Teorema 4.0.7. Sejam $W$ um subconjunto finito e não-vazio de $S^{m-1}, \lambda \in(-1,1)$ e $\rho \in \mathcal{O}_{m}$. Assuma que $\varepsilon$ possui alguma componente igual a 1. Se $W$ é um q-design e $W_{\lambda}^{\prime \prime}$ é um $q_{1}$-design em $S^{m-2}$, então

$$
W_{\rho}=\left(W \backslash W_{\lambda}\right) \cup \rho\left(W_{\lambda}\right)
$$

é um $q \wedge q_{1}$-design.

Prova: É semelhante à prova do Teorema 4.0.6. Entretanto a aplicação biunívoca daquela prova tem que ser substituída pela aplicação biunívoca $x_{\varepsilon}^{\prime} \in W_{\lambda, \varepsilon}^{\prime} \rightarrow x^{\prime \prime} \in S^{m-2}$, onde $x^{\prime \prime}$ é obtido de $x_{\varepsilon}^{\prime}$ excluindo-se sua coordenada nula correspondente à coordenada não-nula de $\varepsilon$.

Exemplo 4.0.1. Sejam $W$ como no Exemplo 2.1.4 e $\varepsilon=(0,0,1) \in \mathbb{R}^{3}$. A rotação $\rho \in \mathcal{O}_{3}$ dada por

$$
\rho(x, y, z)=\left(\begin{array}{ccc}
\cos \theta & \sin \theta & 0 \\
-\sin \theta & \cos \theta & 0 \\
0 & 0 & 1
\end{array}\right)\left(\begin{array}{l}
x \\
y \\
z
\end{array}\right),
$$

satisfaz $\rho(\varepsilon)=\varepsilon$. Se $\lambda=0$ então $W_{\lambda}=W_{\lambda, \varepsilon}^{\prime}=\{(1,0,0),(0,1,0),(-1,0,0),(0,-1,0)\}$ e $W_{\lambda}^{\prime \prime}=\{(1,0),(0,1),(-1,0),(0,-1)\}$. O Exemplo 2.1 .3 revela que $W_{\lambda}^{\prime \prime}$ é um 3-design em 
$S^{1}$ enquanto que um argumento similar àquele do Exemplo 2.1.4 revela que $W$ é um 3design em $S^{2}$. Logo, o Teorema 4.0.7 é aplicável. Em particular, quando $\theta=\pi / 4$ podemos concluir que

$$
W_{\rho}=\{(0,0,1),(0,0,-1)\} \cup\{(\sqrt{2} / 2,0,0),(0, \sqrt{2} / 2,0),(-\sqrt{2} / 2,0,0),(0,-\sqrt{2} / 2,0)\}
$$

é um 3-design em $S^{2}$.

O resultado a seguir é uma generalização do Teorema 4.0.6, onde para $\lambda \in(-1,1)$ consideramos o conjunto

$$
W_{-\lambda}:=W \cap\left\{x \in S^{m-1}: x \cdot \varepsilon=-\lambda\right\}
$$

Teorema 4.0.8. Sejam $W$ um subconjunto finito e não-vazio de $S^{m-1}$ e $\lambda \in(-1,1)$. Seja $\rho \in \mathcal{O}_{m} \operatorname{com} \rho(\varepsilon)=\varepsilon$. Se $W$ é um q-design, $W_{\lambda, \varepsilon}^{\prime}$ e $W_{-\lambda, \varepsilon}^{\prime}$ são $\left(q_{1}-1\right)$-designs e $W_{\lambda, \varepsilon}^{\prime} \cup W_{-\lambda, \varepsilon}^{\prime}$ é um $q_{1}$-design então

$$
W_{\rho}=\left(W \backslash\left(W_{\lambda} \cup W_{-\lambda}\right)\right) \cup \rho\left(W_{\lambda} \cup W_{-\lambda}\right)
$$

é um $q_{1}$-design.

Prova: É muito parecida com a prova do Teorema 4.0.6. Aqui, a essência da prova consiste em verificar que a soma

$$
\sum_{x \in \rho\left(W_{\lambda}\right)} R_{n}^{m}(x \cdot y)+\sum_{x \in \rho\left(W_{-\lambda}\right)} R_{n}^{m}(x \cdot y), \quad y \in S^{m-1}
$$

independe de $\rho$ quando $n \in\left\{1,2, \ldots, q_{1}\right\}$, o que pode ser feito da mesma forma. 


\section{Apêndice 1}

O objetivo do Apêncice é justificar a formação do conjunto

$$
B_{\lfloor q / 2\rfloor}:=\left\{\mathcal{G}_{\lfloor q / 2\rfloor}(x \cdot y): x \in W\right\},
$$

utilizado na prova do Teorema 3.0.2.

Seja $k \in\{0,1, \ldots, q\}$ e considere o conjunto $B_{k}=\left\{\mathcal{G}_{k}(x \cdot \xi): x \in W\right\}$. Na aplicação do Teorema 2.4.2, temos que considerar a variação de índices determinada pela desigualdade $0 \leq k \leq l \leq q-k$. A tabela abaixo ilustra as possibilidades:

\begin{tabular}{|c|c|c|}
\hline$k$ & $l$ & $q-k$ \\
\hline \hline 0 & $0,1, \ldots, q$ & $q$ \\
\hline 1 & $1,2, \ldots, q-1$ & $q-1$ \\
\hline$\vdots$ & $\vdots$ & $\vdots$ \\
\hline$\lfloor q / 2\rfloor-1$ & $\lfloor q / 2\rfloor-1,\lfloor q / 2\rfloor,\lfloor q / 2\rfloor+1$ & $\lfloor q / 2\rfloor+1$ \\
\hline$\lfloor q / 2\rfloor$ & $\lfloor q / 2\rfloor$ & $\lfloor q / 2\rfloor$ \\
\hline
\end{tabular}

Quando da utilização do Teorema 2.4.2 na prova dos resultados do Capítulo 3, a maximização de $l$ foi crucial. Logo, consultando-se a tabela, vemos que $k=\lfloor q / 2\rfloor$ produz a "melhor" informação, ou seja, que

$$
f(y)=|W|^{-1} \sum_{x \in W} f(x) \mathcal{G}_{\lfloor q / 2\rfloor}(x \cdot y), \quad y \in S^{m-1},
$$

quando $f \in \mathcal{P}_{\lfloor q / 2\rfloor}$. 


\title{
Notações
}

\author{
$S^{m-1} \quad$ Esfera unitária de $\mathbb{R}^{m}$; \\ $[X] \quad$ Espaço gerado pelo conjunto $X$; \\ $\sigma_{m-1} \quad$ Medida na esfera unitária de $\mathbb{R}^{m}$, isto é, $\sigma_{m-1}=\int_{S^{m-1}} d \sigma$; \\ * Variável não explicitada; \\ $L^{2}\left(S^{m-1}\right)$ Espaço vetorial das funções de quadrado $\sigma_{m-1}$-integrável em $S^{m-1}$; \\ $\|\cdot\| \quad$ Norma usual do $\mathbb{R}^{m}$; \\ $\delta_{i, j} \quad$ Delta de kronecker usual associado aos índices $i$ e $j$; \\ $\mathbb{Z}_{+} \quad$ Conjunto dos inteiros não negativos; \\ - $\quad$ Produto interno de $\mathbb{R}^{m}$; \\ $|W| \quad$ Cardinalidade do conjunto $W$; \\ $P_{n}^{(\alpha, \beta)} \quad$ Polinômio de Jacobi de grau $n$ e parâmetros $\alpha$ e $\beta$ \\ $\Pi_{n} \quad$ Conjunto dos polinômios de grau no máximo $n$ em $m$ variáveis; \\ $\mathcal{O}_{m} \quad$ Conjunto do operadores ortogonais sobre $\mathbb{R}^{m}$ \\ $\rho^{*} \quad$ Operador adjunto do operador $\rho \in \mathcal{O}(m)$; \\ $I_{m} \quad$ Matriz identidade de ordem $m$; \\ $a \wedge b \quad$ O menor dos números $a$ e $b, ;$ \\ $\lfloor x\rfloor \quad$ Menor inteiro menor ou igual a $x$; \\ l.i. Linearmente independente; \\ U் União disjunta; \\ $S_{\varepsilon}^{m-1} \quad$ Equador de $S^{m-1}$ que é perpendicular a $\varepsilon$.
}




\section{Referências Bibliográficas}

[1] Askey, R. Orthogonal polynomials and special functions, Philadelphia : Society for Industrial and Applied Mathematics, 1975, Regional conference series in applied mathematics : 21, 1974.

[2] Axler, S.; Bourdon, P.; Ramey, W. Harmonic function theory, Springer- Verlag, NY, 1992.

[3] Bajnok, B.; Construction of designs on the 2-sphere. European J. Combin. 12 (1991), no. 5 , p. $377-382$.

[4] Bingham, B. J. C., Positive definite function on spheres, Proc. Camb. Phil. Soc. 73, (1973), p. 145-156.

[5] Boyvalenkov, P.; Danev, D.; Nikova, S.; Nonexistence of certain spherical designs of odd strengths and cardinalities. Discrete Comput. Geom. 21 (1999), no. 1, p. 143-156.

[6] Boyvalenkov, P.; Nikova, Svetla Improvements of the lower bounds on the size of some spherical designs. Math. Balkanica (N.S.) 12 (1998), no. 1-2, p. 151-160.

[7] Carlitz, L., The products of two ultraspherical polinomials, Proc. Glasgow Math. assoc., 5 (1961-2), p. 76-79

[8] Delsarte, P.; Goethals, J. M.; Seidel, J. J. Spherical codes and designs. Geometriae Dedicata 6 (1977), no. 3, p. 363-388.

[9] Goethals, J.-M.; Seidel, J. J.- Spherical designs. Relations between combinatorics and other parts of mathematics (Proc. Sympos. Pure Math., Ohio State Univ., Columbus, Ohio, 1978), pp. 255-272, Proc. Sympos. Pure Math., XXXIV, Amer. Math. Soc., Providence, R.I., 1979. 
[10] Goethals, J. M.; Seidel, J. J.; The football. Nieuw Arch. Wisk. (3) 29 (1981), no. 1, p. $50-58$.

[11] Groemer, H., Geometric applications of Fourier series and Spherical harmonics, Cambridge university press, 1996.

[12] Hylleraas, E., Linearization of products of Jacobi polinomials, Math. Scand., 10 (1962), p. 189-200.

[13] Menegatto, V. A., Tese de doutorado: interpolation on spherical spaces, University of Texas at Austin, 1992.

[14] Morimoto, M. Analytic functionals on the sphere. Amer. Math. Soc., Providence, 1998.

[15] Müller, C. Analysis os spherical symmetries in euclidean spaces, Applied Mathematical Sciences, Vol. 296, Springer- Verlag, NY, 1998.

[16] Piantella, A. C. Dissertação de mestrado: Interpolação e hiperinterpolação em esferas, ICMC/ Universidade de São Paulo, 2003.

[17] Rainville, E. D. - Special functions. Reprint of 1960 first edition. Chelsea Publishing Co., Bronx, N.Y., 1971.

[18] Reimer, M., Constructive theory of multivariate functions with an application to tomography, Bibliographisches Institut, Mannheim, 1990.

[19] Reimer, M., Multivariate Polynomial Approximation, Birkhäuser Verlag, ISNM, vol. 144.

[20] Schoenberg, I. J., Positive definite functions on spheres. Duke Math. J. 9, (1942). 96 $-108$.

[21] Stein, Elias M.; Weiss, Guido Introduction to Fourier analysis on Euclidean spaces. Princeton Mathematical Series, No. 32. Princeton University Press, Princeton, N.J., 1971.

[22] Szegö, G. Orthogonal polinomials, Amer. Math. Soc., Providence, 1939. 
[23] Yudin, V. A. Rotation of spherical designs. (Russian) Problemy Peredachi Informatsii 36 (2000), no. 3, p. 39-45. 


\section{Índice Remissivo}

Design antipodal, 34

Designs esféricos, 15

Dimensão

de $\mathcal{A}_{n}, 5$

de $\mathcal{H}_{n}, 5$

de $\mathcal{P}_{n}, 5$

Fórmula

da adição, 6

da adição para polinômios de Gegen-

bauer, 11

de Dougall, 11

de Funk-Kecke, 8

Forma quadrádica, 6

Harmônicos

esféricos, 3

zonais, 6

Núcleo de reprodução, 12

Polinômios de Legendre, 9

Rotação de Designs, 37

Sistema fundamental, 7 